\title{
Global analysis of a multi-group SIR epidemic model with nonlinear incidence rates and distributed moving delays between patches
}

\author{
Yoshiaki Muroya $^{1}$, Toshikazu Kuniya ${ }^{2}$ and Yoichi Enatsu ${ }^{\bowtie 3}$ \\ ${ }^{1}$ Department of Mathematics, Waseda University, 3-4-1 Ohkubo, Shinjuku-ku, Tokyo 169-8555, Japan \\ ${ }^{2}$ Graduate School of System Informatics, Kobe University, \\ 1-1 Rokkodai-cho, Nada-ku, Kobe 657-8501, Japan \\ ${ }^{3}$ Department of Mathematical Information Science, Faculty of Science, Tokyo University of Science, \\ 1-3 Kagurazaka, Shinjuku-ku, Tokyo 162-8601, Japan
}

Appeared 11 August 2016

Communicated by Tibor Krisztin

\begin{abstract}
In this paper, applying Lyapunov functional approach, we establish sufficient conditions under which each equilibrium is globally asymptotically stable for a class of multi-group SIR epidemic models. The incidence rate is given by nonlinear incidence rates and distributed delays incorporating not only an exchange of individuals between patches through migration but also cross patch infection between different groups. We show that nonlinear incidence rates and distributed delays have no influence on the global stability, but patch structure has. Moreover, the present results generalize known results on the global stability of a heroin model with two delays considered in the recent literatures. We also offer new techniques to prove the boundedness of the solutions, the existence of the endemic equilibrium and permanence of the model.
\end{abstract}

Keywords: heroin model, multi-group SIR epidemic model, patch, global stability, Lyapunov function.

2010 Mathematics Subject Classification: 34K20, 34K25, 92D30.

\section{Introduction}

Due to the recent development of qualitative and quantitative analysis for disease transmission models, mathematical models have widely been applied to investigate the spread of habituation of getting addicted to drugs such as heroin (see e.g., [7, 12, 17, 22, 28, 34,35]).

Dividing the host population into three compartments; susceptible individuals $S(t)$, heroin users $U_{1}(t)$ and heroin users undergoing treatment $U_{2}(t)$, White and Comiskey [35] investi-

\footnotetext{
${ }^{\bowtie}$ Corresponding author. Email: yenatsu@rs.tus.ac.jp
} 
gated the following heroin model:

$$
\left\{\begin{array}{l}
S^{\prime}(t)=b-\frac{\beta S(t) U_{1}(t)}{N(t)}-d S(t), \\
U_{1}^{\prime}(t)=\frac{\beta S(t) U_{1}(t)}{N(t)}-\gamma U_{1}(t)+\frac{\kappa U_{1}(t) U_{2}(t)}{N(t)}-(d+\varepsilon) U_{1}(t), \\
U_{2}^{\prime}(t)=\gamma U_{1}(t)-\frac{\kappa U_{1}(t) U_{2}(t)}{N(t)}-(d+\delta) U_{2}(t) .
\end{array}\right.
$$

Here $b$ denotes the birth rate at which individuals in the general population enter the susceptible population. $d$ denotes the death rate from natural causes. It is assumed that new infection of a drug user arises through a standard incidence function, $\beta S(t) U_{1}(t) / N(t)$ (resp. $\kappa U_{1}(t) U_{2}(t) / N(t)$ ) with $\beta$ (resp. $\kappa$ ) denoting the probability for susceptible individuals (resp. drug users in treatment relapsing to untreated use) to be drug users not in treatment. Here drug users in treatment are not assumed to be susceptible again after they quit using drugs. $\gamma$ denotes the rate at which drug users undertake treatment and $\varepsilon$ represents a removal rate of drug users not in treatment, a sum of drug-related deaths rate and a spontaneous recovery rate. $\delta$ is a removal rate of drug users in treatment, a sum of drug-related death rate and a rate of successful "care" for drug user to be in a drug-free recovery. All the parameters are assumed to be positive.

In addition to stability analysis for a drug-free equilibrium [35, Section 3], the stability analysis for a unique endemic equilibrium is achieved by Mulone and Straughan [22] when $\kappa<\beta$. Later, Wang et al. [34] has formulated the model incorporating the mass action incidence rate and established the global stability of the drug-free equilibrium and the unique endemic equilibrium by means of the second compound matrix and under some conditions. These systems are extended to a non-autonomous model by Samanta [28], proving that there exists a unique positive periodic solution which is globally asymptotically stable by a direct Lyapunov method.

Recently, Liu and Zhang [17] introduced distributed delays in the relapse term into a heroin epidemic model without delays. Constructing a proper Lyapunov function, Huang and Liu [12] established the global stability for the heroin epidemic model with a distributed delay $\int_{0}^{h} \tilde{f}(\tau) \gamma U_{1}(t-\tau) e^{-(d+\delta) s} d s$ in place of the term $\kappa U_{1}(t) U_{2}(t) / N(t)$ in the third equation of the model (1.1).

Compared to the above "heavy drug" epidemic model with varying total population size incorporating drug-related deaths as the model (1.1), Muroya et al. [25] considered the following disease "light drug" epidemic model with "not varying total population size" eventually and such that there are no drug-related deaths of the light drug users who are not in treatment and in treatment.

$$
\left\{\begin{array}{l}
S^{\prime}(t)=b-\frac{\beta S(t) U_{1}(t)}{N(t)}-d S(t)+\varepsilon U_{1}(t)+\delta U_{2}(t), \\
U_{1}^{\prime}(t)=\frac{\beta S(t) U_{1}(t)}{N(t)}-\gamma U_{1}(t)+\sigma U_{2}(t)-(d+\varepsilon) U_{1}(t), \\
U_{2}^{\prime}(t)=\gamma U_{1}(t)-\sigma U_{2}(t)-(d+\delta) U_{2}(t) .
\end{array}\right.
$$

In this case, we have $\lim _{t \rightarrow+\infty}\left(S(t)+U_{1}(t)+U_{2}(t)\right)=b / d$. This also implies that the total population size does not eventually vary oscillatory but converges to a positive constant $b / d$.

On the other hand, Guo et al. [8] have first succeeded in the proof of global stability for a multi-group SIR epidemic model by making use of the theory of non-negative matrices, Lyapunov functions and a subtle grouping technique in estimating the derivatives of Lyapunov 
functions guided by graph theory. To analyze the global stability of various multi-group epidemic models, many authors literature on multi-group models follow to use this graph theoretic approach (see for example, [4,10,15,16, 29,30,36,37]).

Recently, there are some interesting papers on construction techniques of Lyapunov functions to prove the global stability of equilibria (see, for e.g., Li et al. [14], Kajiwara et al. [20] and Vargas-De-León [32]). Guo et al. [9,11] considered the stage-progression models for HIV / AIDS with amelioration. Li et al. [14] established the global stability of a class of epidemic models by using quite interesting approach, and Muroya et al. [24] generalized their method.

Multi-group epidemic models have played a crucial role to clarify one of the important problems; transportation affects on the spreading pattern of the global pandemic of diseases such as heroin (see for example, Arino [1], Bartlett [2] for a population movement among different groups, Liu and Takeuchi [19], Liu and Zhou [18] and Nakata [26] for the effect of transport-related infection with entry screening, Wang and Zhao [33] for an epidemic model in a patchy environment). In particular, Muroya et al. [24] established general sufficient conditions of the global stability for a multi-group SIR epidemic model with patch structure which takes into account not only an exchange of individuals between patches through migration but also cross patch infection between different groups.

Recently, Fang et al. [7] presented the following heroin epidemic model with two distributed delays and establish the global asymptotic stability.

$$
\begin{aligned}
& S^{\prime}(t)=\Lambda-\beta S(t) \int_{0}^{h_{1}} \tilde{f}(\tau) U_{1}(t-\tau) e^{-\left(\mu+\delta_{1}+p\right) \tau} d \tau-\mu S(t), \\
& U_{1}^{\prime}(t)=\beta S(t) \int_{0}^{h_{1}} \tilde{f}(\tau) U_{1}(t-\tau) e^{-\left(\mu+\delta_{1}+p\right) \tau} d \tau+p \int_{0}^{h_{2}} \tilde{g}(\tau) U_{1}(t-\tau) e^{-\left(\mu+\delta_{1}+p\right) \tau} d \tau, \\
& U_{2}^{\prime}(t)=p U_{1}(t)-\left(\mu+\delta_{2}\right) U_{2}(t)-p \int_{0}^{h_{2}} \tilde{g}(\tau) U_{1}(t-\tau) e^{-\left(\mu+\delta_{1}+p\right) \mu} d \tau,
\end{aligned}
$$

where $\int_{0}^{h_{1}} \tilde{f}(\tau) d \tau=1$ and $\int_{0}^{h_{2}} \tilde{g}(\tau) d \tau=1$. Motivated by Fang et al. [7], in this paper, we aim to investigate the global dynamics of a multi-group epidemic model related to heroin model with nonlinear incidence rates and distributed delays. In the formulation of the model, we divide each population into $n \in \mathbb{N}$ groups and use the following notations (in what follows, $k$ and $j$ belong to $\{1,2, \ldots, n\})$ :

- $S_{k}(t)$ : the number of susceptible individuals in city $k$ at time $t$;

- $I_{k}(t)$ : the number of infected individuals (heroin users) in city $k$ at time $t$;

- $R_{k}(t)$ : the number of recovered individuals (heroin users under treatment) in city $k$ at time $t$;

- $b_{k}$ : the recruitment rate of the population in city $k$;

- $\mu_{k i}$ : the natural death rates of susceptible $(i=1)$, infected $(i=2)$ and recovered $(i=3)$ individuals in city $k$, respectively, satisfying

$$
\mu_{k 1} \leq \min \left(\mu_{k 2}, \mu_{k 3}\right) \quad \text { for any } \quad k=1,2, \ldots, n \text {; }
$$

- $\beta_{k j}$ : the transmission parameter between the susceptible individuals in city $k$ and the infected individuals in city $j$;

- $\gamma_{k}$ : the recovery rate of the infected individuals in city $k$; 
- $l_{k j}$ : the per capita rate at which the susceptible individuals in city $j$ leave toward city $k$ $\left(l_{k k}=0\right)$;

- $m_{k j}$ : the per capita rate at which the infected individuals in city $j$ leave toward city $k$ $\left(m_{k k}=0\right)$;

- $n_{k j}$ : the per capita rate at which the recovered individuals in city $j$ leave toward city $k$;

- $f_{k j}(\tau)\left(0 \leq \tau \leq h_{1}\right)$ : the distribution kernel for the time delay of infection such that $\int_{0}^{h_{1}} f_{k j}(\tau) d \tau=1$;

- $g_{k j}(\sigma)\left(0 \leq \sigma \leq h_{2}\right)$ : the distribution kernel for the time delay of movement such that $\int_{0}^{h_{2}} g_{k j}(\sigma) d \sigma=1$;

- $p_{k}(\sigma)\left(0 \leq \sigma \leq h_{3}\right)$ : the distribution kernel for the time delay for which a heroin user under treatment returns to untreated user after cessation of a drug treatment program such that $\int_{0}^{h_{3}} p_{k}(\sigma) d \sigma=1$.

In what follows, we assume that $g_{k j}(\sigma)=0$ and $p_{k}(\sigma)=0$ outside of each domain. Then, putting $\max \left(h_{2}, h_{3}\right)$ as $h_{2}$ again, we have that $\int_{0}^{h_{2}} g_{k j}(\sigma) d \sigma=\int_{0}^{h_{2}} p_{k}(\sigma) d \sigma=1$. Moreover, we use a locally Lipschitz continuous function $G(I)$ on $[0,+\infty)$ such that

$$
\left\{\begin{array}{l}
\text { there exists some sufficiently large positive constant } b \text { such that } G(I) \text { is monotone } \\
\text { increasing on }[0, b] \text { and } I / G(I) \text { is monotone increasing on }(0,+\infty) \text { and } \\
\lim _{I \rightarrow+0}(I / G(I))=1 .
\end{array}\right.
$$

Notice that from the condition (1.3), one can see that $G(I) \leq I$ for $I>0$ and $G(0)=0$. Using these parameters, we formulate the following multi-group SIR epidemic model with nonlinear incidence rates and distributed delays, which is related to the heroin model:

$$
\left\{\begin{aligned}
\frac{d S_{k}(t)}{d t}= & b_{k}-\mu_{k 1} S_{k}(t)-S_{k}(t) \sum_{j=1}^{n} \beta_{k j} \int_{0}^{h_{1}} f_{k j}(\tau) G\left(I_{j}(t-\tau)\right) d \tau \\
& +\sum_{j=1}^{n}\left(l_{k j} \int_{0}^{h_{2}} g_{k j}(\sigma) S_{j}(t-\sigma) d \sigma-l_{j k} S_{k}(t)\right) \\
\frac{d I_{k}(t)}{d t}= & S_{k}(t)\left(\sum_{j=1}^{n} \beta_{k j} \int_{0}^{h_{1}} f_{k j}(\tau) G\left(I_{j}(t-\tau)\right) d \tau\right)-\left(\mu_{k 2}+\gamma_{k}\right) I_{k}(t) \\
& +\gamma_{k} \int_{0}^{h_{2}} p_{k}(\sigma) e^{-\mu_{k 3} \sigma} I_{k}(t-\sigma) d \sigma \\
& +\sum_{j=1}^{n}\left(m_{k j} \int_{0}^{h_{2}} g_{k j}(\sigma) I_{j}(t-\sigma) d \sigma-m_{j k} I_{k}(t)\right), \\
\frac{d R_{k}(t)}{d t}= & \gamma_{k} I_{k}(t)-\gamma_{k} \int_{0}^{h_{2}} p_{k}(\sigma) e^{-\mu_{k 3} \sigma} I_{k}(t-\sigma) d \sigma-\mu_{k 3} R_{k}(t) \\
& +\sum_{j=1}^{n}\left(n_{k j} \int_{0}^{h_{2}} g_{k j}(\sigma) R_{j}(t-\sigma) d \sigma-n_{j k} R_{k}(t)\right), \quad k=1,2, \ldots, n .
\end{aligned}\right.
$$

In the model (1.4), the number of newly infected individuals in city $k$ is given by a sum of nonlinear incidence rates $\beta_{k j} S_{k}(t) \int_{0}^{h_{1}} f_{k j}(\tau) G\left(I_{j}(t-\tau)\right) d \tau$ for $k, j=1,2, \ldots, n$. One can 
see that the term $\beta_{k j} S_{k}(t) \int_{0}^{h_{1}} f_{k j}(\tau) G\left(I_{j}(t-\tau)\right) d \tau$ with $k \neq j$ describes the effect of cross patch infection between groups $k$ and $j, j \neq k$ who travel shortly from other city $j$ into city $k$ with a time delay $\tau \in\left[0, h_{1}\right]$. On the other hand, the term $\sum_{j=1}^{n} l_{k j} \int_{0}^{h_{2}} g_{k j}(\sigma) S_{j}(t-\sigma) d \sigma$ (resp. $\sum_{j=1}^{n} m_{k j} \int_{0}^{h_{2}} g_{k j}(\sigma) I_{j}(t-\sigma) d \sigma$ ) describes the inflow of susceptible individuals (resp. infected individuals) from all other cities $j$ into city $k$ at time $t$. The term $\sum_{j=1}^{n} l_{j k} S_{k}(t)$ (resp. $\left.\sum_{j=1}^{n} m_{j k} I_{k}(t)\right)$ is the outflow of susceptible individuals (resp. infected individuals) from city $k$ towards all other cities $j$. Once an individual in patch $j$ moves to patch $k$, then the individual homogeneously mixes with individuals in patch $k$ and is counted as an individual in patch $k$ since there is no track for each individual. By regarding $I_{k}$ as the number of heroin users and $R_{k}$ as that of heroin users under treatment, the model (1.4) can be interpreted as the heroin model. In the model (1.4), as in Fang et al. [7], we assume that the heroin users under treatment can return to untreated users depending on their different characters and external influences. Such difference can be taken into account by the time delay modulated by the distribution kernel $p_{k}(\sigma)$.

Since the first two equations in system (1.4) do not contain the variable $R_{k}, k=1,2, \ldots, n$, it is equivalent to

$$
\left\{\begin{aligned}
\frac{d S_{k}(t)}{d t}= & b_{k}-\mu_{k 1} S_{k}(t)-S_{k}(t) \sum_{j=1}^{n} \beta_{k j} \int_{0}^{h_{1}} f_{k j}(\tau) G\left(I_{j}(t-\tau)\right) d \tau \\
& +\sum_{j=1}^{n}\left(l_{k j} \int_{0}^{h_{2}} g_{k j}(\sigma) S_{j}(t-\sigma) d \sigma-l_{j k} S_{k}(t)\right) \\
\frac{d I_{k}(t)}{d t}= & S_{k}(t)\left(\sum_{j=1}^{n} \beta_{k j} \int_{0}^{h_{1}} f_{k j}(\tau) G\left(I_{j}(t-\tau)\right) d \tau\right)-\left(\mu_{k 2}+\gamma_{k}\right) I_{k}(t) \\
& +\gamma_{k} \int_{0}^{h_{2}} p_{k}(\sigma) e^{-\mu_{k 3} \sigma} I_{k}(t-\sigma) d \sigma \\
& +\sum_{j=1}^{n}\left(m_{k j} \int_{0}^{h_{2}} g_{k j}(\sigma) I_{j}(t-\sigma) d \sigma-m_{j k} I_{k}(t)\right), \quad k=1,2, \ldots, n .
\end{aligned}\right.
$$

Let

$$
\kappa_{k}=\gamma_{k} \int_{0}^{h_{2}} p_{k}(\sigma) e^{-\mu_{k 3} \sigma} d \sigma\left(<\gamma_{k}\right), \quad \tilde{g}_{k k}(\sigma)=\frac{p_{k}(\sigma) e^{-\mu_{k 3} \sigma}}{\int_{0}^{h_{2}} p_{k}(\sigma) e^{-\mu_{k 3} \sigma} d \sigma}, \quad \tilde{\gamma}_{k}=\gamma_{k}-\kappa_{k}(>0) .
$$

Then, the second equation in (1.5) becomes

$$
\begin{aligned}
\frac{d I_{k}(t)}{d t}= & S_{k}(t)\left(\sum_{j=1}^{n} \beta_{k j} \int_{0}^{h_{1}} f_{k j}(\tau) G\left(I_{j}(t-\tau)\right) d \tau\right)-\left(\mu_{k 2}+\tilde{\gamma}_{k}\right) I_{k}(t) \\
& -\kappa_{k} I_{k}(t)+\kappa_{k} \int_{0}^{h_{2}} \tilde{g}_{k k}(\sigma) I_{k}(t-\sigma) d \sigma \\
& +\sum_{j=1, j \neq k}^{n}\left(m_{k j} \int_{0}^{h_{2}} g_{k j}(\sigma) I_{j}(t-\sigma) d \sigma-m_{j k} I_{k}(t)\right), \quad k=1,2, \ldots, n .
\end{aligned}
$$

Without loss of generality, we can regard $\kappa_{k}$ as $m_{k k}, \tilde{g}_{k k}(\sigma)$ as $g_{k k}(\sigma)$ and since $m_{k k}=0$ in original and $\int_{0}^{h_{2}} \tilde{g}_{k k}(\sigma) d \sigma=1$. Furthermore, for simplicity, we omit the notation ${ }^{\sim}$ from $\tilde{\gamma}_{k}$. 
Then, we arrive at the following main form of our model:

$$
\left\{\begin{aligned}
\frac{d S_{k}(t)}{d t}= & b_{k}-\mu_{k 1} S_{k}(t)-S_{k}(t) \sum_{j=1}^{n} \beta_{k j} \int_{0}^{h_{1}} f_{k j}(\tau) G\left(I_{j}(t-\tau)\right) d \tau \\
& +\sum_{j=1}^{n}\left(l_{k j} \int_{0}^{h_{2}} g_{k j}(\sigma) S_{j}(t-\sigma) d \sigma-l_{j k} S_{k}(t)\right), \\
\frac{d I_{k}(t)}{d t}= & S_{k}(t)\left(\sum_{j=1}^{n} \beta_{k j} \int_{0}^{h_{1}} f_{k j}(\tau) G\left(I_{j}(t-\tau)\right) d \tau\right)-\left(\mu_{k 2}+\gamma_{k}\right) I_{k}(t) \\
& +\sum_{j=1}^{n}\left(m_{k j} \int_{0}^{h_{2}} g_{k j}(\sigma) I_{j}(t-\sigma) d \sigma-m_{j k} I_{k}(t)\right), \quad k=1,2, \ldots, n
\end{aligned}\right.
$$

Note that (1.6) is an extended model of that in Muroya et al. [24] in that nonlinear incidence rates with delays are incorporated.

The initial conditions of system (1.6) take the form

$$
S_{k, 0}=\phi_{1}^{k} \in C\left(\left[-h_{2}, 0\right], \mathbb{R}_{+}\right), \quad I_{k, 0}=\phi_{2}^{k} \in C\left([-h, 0], \mathbb{R}_{+}\right), \quad h=\max \left(h_{1}, h_{2}\right), \quad k=1,2, \ldots, n .
$$

Moreover, we assume that

$$
\text { the } n \times n \text { matrix } \mathbf{B}=\left[\beta_{k j}\right]_{n \times n} \text { is irreducible, }
$$

and there exists a positive vector $\left(c_{1}, c_{2}, \ldots, c_{n}\right)$ such that

$$
c_{k} \mu_{k 1}+\sum_{j=1}^{n}\left(l_{j k} c_{k}-l_{k j} c_{j}\right)>0, \quad k=1,2, \ldots, n
$$

The last condition (1.9) is used to guarantee the boundedness of the solutions of (1.6) for the delayed terms of patch structure in the proof of Lemma 2.1 (cf. Muroya et al. [24]). For example, if $\mu_{k 1}+\sum_{j=1}^{n}\left(l_{j k}-l_{k j}\right)>0, k=1,2, \ldots, n$, then (1.9) is satisfied.

Put

$$
\tilde{l}_{k k}=\sum_{j=1}^{n}\left(1-\delta_{j k}\right) l_{j k}, \quad \tilde{m}_{k k}=\sum_{j=1}^{n}\left(1-\delta_{j k}\right) m_{j k}, \quad \delta_{k j}=\left\{\begin{array}{ll}
1, & \text { if } k=j, \\
0, & \text { if } k \neq j,
\end{array} \quad k=1,2, \ldots, n .\right.
$$

From (1.8), one can see that $\sum_{j=1}^{n}\left(1-\delta_{k j}\right) \beta_{k j}+\tilde{m}_{k k}>0, k=1,2, \ldots, n$. Let $\mathbf{H}$ and $\mathbf{b}$ be an $n \times n$ matrix and a positive $n$-column vector defined by

$$
\mathbf{H}=\left[\begin{array}{cccc}
\mu_{11}+\tilde{l}_{11} & -l_{12} & \cdots & -l_{1 n} \\
-l_{21} & \mu_{21}+\tilde{l}_{22} & \cdots & -l_{2 n} \\
\vdots & \vdots & \ddots & \vdots \\
-l_{n 1} & -l_{n 2} & \cdots & \mu_{n 1}+\tilde{l}_{n n}
\end{array}\right] \text { and } \quad \mathbf{b}=\left[\begin{array}{c}
b_{1} \\
b_{2} \\
\vdots \\
b_{n}
\end{array}\right]
$$

and $\mathbf{S}^{0}=\left(S_{1}^{0}, S_{2}^{0}, \ldots, S_{n}^{0}\right)^{T}$ be the positive $n$-column vector such that

$$
\mathbf{S}^{0}=\mathbf{H}^{-1} \mathbf{b}
$$

By (1.10), $\mathbf{H}$ is an $M$-matrix (see, e.g., Berman and Plemmons [3] or Varga [31]), and $\mathbf{S}^{0}$ depends on $l_{k j}, k, j=1,2, \ldots, n$. 
For $\mathbf{S}=\left(S_{1}, S_{2}, \cdots, S_{n}\right)^{T}$ and $\mathbf{S}^{0}=\left(S_{1}^{0}, S_{2}^{0}, \cdots, S_{n}^{0}\right)^{T}$ defined by (1.12), let $\tilde{\mathbf{V}}$ be an $n \times n$ matrix such that

$$
\tilde{\mathbf{V}}=\left[\begin{array}{cccc}
\tilde{V}_{1} & 0 & \cdots & 0 \\
0 & \tilde{V}_{2} & \cdots & 0 \\
\vdots & \vdots & \ddots & \vdots \\
0 & 0 & \cdots & \tilde{V}_{n}
\end{array}\right], \quad \tilde{V}_{k}=\mu_{k 2}+\gamma_{k}+\tilde{m}_{k k}, k=1,2, \ldots, n,
$$

and $\tilde{\mathbf{F}}(\mathbf{S})$ be an $n \times n$ matrix such that

$$
\begin{aligned}
\tilde{\mathbf{F}}(\mathbf{S}) & =\left[\begin{array}{cccc}
\tilde{F}_{11}\left(S_{1}\right) & \tilde{F}_{12}\left(S_{1}\right) & \cdots & \tilde{F}_{1 n}\left(S_{1}\right) \\
\tilde{F}_{21}\left(S_{2}\right) & \tilde{F}_{22}\left(S_{2}\right) & \cdots & \tilde{F}_{2 n}\left(S_{2}\right) \\
\vdots & \vdots & \ddots & \vdots \\
\tilde{F}_{n 1}\left(S_{n}\right) & \tilde{F}_{n 2}\left(S_{n}\right) & \cdots & \tilde{F}_{n n}\left(S_{n}\right)
\end{array}\right]=\left[\tilde{F}_{k j}\left(S_{k}\right)\right]_{n \times n}, \\
\tilde{F}_{k j}\left(S_{k}\right) & =\left\{\begin{array}{lll}
S_{k} \beta_{k j}, & k=j \\
S_{k} \beta_{k j}+m_{k j}, & k \neq j .
\end{array}\right.
\end{aligned}
$$

Let us also define $\tilde{\mathbf{M}}(\mathbf{S})$ to be an $n \times n$ matrix such that

$$
\tilde{\mathbf{M}}(\mathbf{S})=\tilde{\mathbf{V}}^{-1} \tilde{\mathbf{F}}(\mathbf{S})=\left[\tilde{M}_{k j}\right]_{n \times n^{\prime}}, \quad \tilde{M}_{k j}=\frac{S_{k} \beta_{k j}+\left(1-\delta_{k j}\right) m_{k j}}{\mu_{k 2}+\gamma_{k}+\tilde{m}_{k k}}, \quad k, j=1,2, \ldots, n .
$$

Let the threshold parameter $\tilde{R}_{0}$ be defined by

$$
\tilde{R}_{0}=\rho\left(\tilde{\mathbf{M}}\left(\mathbf{S}^{0}\right)\right) .
$$

It is easy to see that $\tilde{R}_{0}$ corresponds to the well-known basic reproduction number $R_{0}$ (see for example, Diekmann and Heesterbeek [5]). We now consider the following set $\Gamma$ defined by

$$
\Gamma=\left\{\left(S_{1}, I_{1}, S_{2}, I_{2}, \ldots, S_{n}, I_{n}\right) \in \mathbb{R}_{+}^{2 n} \mid S_{k} \leq S_{k}^{0}, S_{k}+I_{k} \leq \bar{N}_{k}^{*}, k=1,2, \ldots, n\right\},
$$

where $\bar{N}_{k}^{*}, k=1,2, \ldots, n$ are the positive solutions of the following system:

$$
\left(\mu_{k 2}+\tilde{m}_{k k}+m_{k k}\right) \bar{N}_{k}^{*}-\sum_{j=1}^{n} m_{k j} \bar{N}_{j}^{*}=\overline{\tilde{b}}_{k \prime} \quad k=1,2, \ldots, n,
$$

and

$$
\overline{\tilde{b}}_{k}:=b_{k}+\max \left\{\left(\mu_{k 2}+\gamma_{k}+\tilde{m}_{k k}\right)-\left(\mu_{k 1}+\tilde{l}_{k k}\right), 0\right\} S_{k}^{0}+\sum_{j=1}^{n} \max \left\{l_{k j}-m_{k j}, 0\right\} S_{j}^{0} .
$$

Since $\Gamma$ is a positive invariant set (see Lemma 2.1) for the solutions of (1.6), to choose $\Gamma$ as the feasible region of (1.6), we need the last part of (1.14) (see the proof of the first part of Theorem 1.1 for $\tilde{R}_{0}<1$ in Section 3). Let $\Gamma^{0}$ be the interior of $\Gamma$.

By (1.8), we have that

$$
\tilde{\mathbf{M}}(\mathbf{S}) \text { is irreducible in } \boldsymbol{\Gamma} \text {. }
$$

In this paper, we establish the global stability for the multi-group SIR model (1.6) with patch structure. This implies that we extend not only the result of Fang et al. [7] for the multi-group heroin model with patch structure, but also the result of Muroya et al. [24] for the model with delays and nonlinear incidence rates. Moreover, we offer new techniques to prove the boundedness of the solutions of (1.6), the existence of the endemic equilibrium and permanence of (1.6).

The main theorem in this paper is as follows. 


\section{Theorem 1.1.}

(i) For $\tilde{R}_{0}<1$, if there exists a positive $n$-column vector $\mathbf{u}=\left(u_{1}, u_{2}, \ldots, u_{n}\right)^{T}$ such that

$$
\left\{\begin{array}{l}
u_{k}\left(\mu_{k 1}+\tilde{l}_{k k}\right)-\sum_{j=1}^{n} u_{j}\left(1-\delta_{j k}\right) l_{j k} \geq 0, \\
u_{k}\left(\mu_{k 2}+\gamma_{k}+\tilde{m}_{k k}\right)-\sum_{j=1}^{n} u_{j}\left\{\beta_{j k} S_{j}^{0}+\left(1-\delta_{j k}\right) m_{j k}\right\}>0, \text { for any } k=1,2, \ldots, n,
\end{array}\right.
$$

then the disease-free equilibrium $\mathbf{E}^{0}=\left(S_{1}^{0}, 0, S_{2}^{0}, 0, \ldots, S_{n}^{0}, 0\right)$ of $(1.6)$ is globally asymptotically stable in $\boldsymbol{\Gamma}$.

(ii) For $\tilde{R}_{0}>1$, system (1.6) is uniformly persistent in $\Gamma^{0}$ and there exists at least one endemic equilibrium $\mathbf{E}^{*}=\left(S_{1}^{*}, I_{1}^{*}, S_{2}^{*}, I_{2}^{*}, \ldots, S_{n}^{*}, I_{n}^{*}\right)$ in $\Gamma^{0}$. Moreover, if there exists a positive $n$-column vector $\mathbf{v}=\left(v_{1}, v_{2}, \ldots, v_{n}\right)^{T}$ such that

$$
\left\{\begin{array}{l}
v_{k}\left(\mu_{k 1}+\tilde{l}_{k k}\right)-\sum_{j=1}^{n} v_{j}\left(1-\delta_{j k}\right) l_{j k} \geq 0 \\
v_{k}\left(\mu_{k 2}+\gamma_{k}+\tilde{m}_{k k}\right)-\sum_{j=1}^{n} v_{j}\left\{\beta_{j k} S_{j}^{*} \frac{G\left(I_{k}^{*}\right)}{I_{k}^{*}}+\left(1-\delta_{j k}\right) m_{j k}\right\} \geq 0, \text { for any } k=1,2, \ldots, n,
\end{array}\right.
$$

then $\mathbf{E}^{*}$ is globally asymptotically stable in $\Gamma^{0}$.

We note that if $S_{k}^{0}=b_{k} / \mu_{k 1}$ of (1.12), for example, $l_{k j}=0$ for any $k \neq j$, then for $\tilde{R}_{0}=1$, we can conclude that the disease-free equilibrium $\mathbf{E}^{0}=\left(S_{1}^{0}, 0, S_{2}^{0}, 0, \ldots, S_{n}^{0}, 0\right)$ of (1.6) is globally asymptotically stable in $\Gamma$, because for $S_{k}^{0}=b_{k} / \mu_{k 1}$ of (1.12), we have that by (1.8), $\rho(\tilde{\mathbf{M}}(\mathbf{S}))<$ $\rho\left(\tilde{\mathbf{M}}\left(\mathbf{S}^{0}\right)\right)$ for any $\mathbf{S}=\left(S_{1}, S_{2}, \ldots, S_{n}\right)$ such that $0<S_{k}<S_{k}^{0}, k=1,2, \ldots, n$. Otherwise, we can not prove the fact that for $\tilde{R}_{0}=1$, the disease-free equilibrium $\mathbf{E}^{0}=\left(S_{1}^{0}, 0, S_{2}^{0}, 0, \ldots, S_{n}^{0}, 0\right)$ of (1.6) is globally asymptotically stable in $\Gamma$ (see Proof of the first part of Theorem 1.1 for $\tilde{R}_{0}<1$ in Section 3).

Corollary 1.2. Assume (1.15) and $\tilde{R}_{0}>1$. Then, there exists positive n-column vector $\mathbf{v}=$ $\left(v_{1}, v_{2}, \ldots, v_{n}\right)^{T}$ such that

$$
\sum_{j=1}^{n} v_{j}\left\{\beta_{j k} S_{j}^{*} \frac{G\left(I_{k}^{*}\right)}{I_{k}^{*}}+\left(1-\delta_{j k}\right) m_{j k}\right\}=v_{k}\left(\mu_{k 2}+\gamma_{k}+\tilde{m}_{k k}\right), \quad k=1,2, \ldots, n,
$$

and for this $\mathbf{v}=\left(v_{1}, v_{2}, \ldots, v_{n}\right)^{T}$, if

$$
v_{k}\left(\mu_{k 1}+\tilde{l}_{k k}\right)-\sum_{j=1}^{n} v_{j}\left(1-\delta_{j k}\right) l_{j k} \geq 0, \quad \text { for any } k=1,2, \ldots, n,
$$

then $\mathbf{E}^{*}$ is globally asymptotically stable in $\boldsymbol{\Gamma}^{0}$.

If there exists a positive $n$-column vector $\mathbf{w}=\left(w_{1}, w_{2}, \ldots, w_{n}\right)$ such that

$$
\sum_{j=1}^{n} w_{j}\left(1-\delta_{j k}\right) l_{j k}=w_{k}\left(\mu_{k 1}+\tilde{l}_{k k}\right), \quad k=1,2, \ldots, n,
$$

and

$$
w_{k}\left(\mu_{k 2}+\gamma_{k}+\tilde{m}_{k k}\right)-\sum_{j=1}^{n} w_{j}\left\{\beta_{j k} S_{j}^{*} \frac{G\left(I_{k}^{*}\right)}{I_{k}^{*}}+\left(1-\delta_{j k}\right) m_{j k}\right\} \geq 0, \quad k=1,2, \ldots, n,
$$

then $\mathbf{E}^{*}$ is globally asymptotically stable in $\Gamma^{0}$. 
The results generalize the known results of a heroin model with two delays considered in the recent literature and imply that nonlinear incidence rates and distributed delays have no influence on the global stability of the model but it depends on patch structure.

The rest of the present paper is organized as follows. In Section 2, we show eventual boundedness of solutions for system (1.6). In Section 3, we prove the global asymptotic stability of the disease-free equilibrium for $\tilde{R}_{0}<1$ (see Theorem 3.1). In Section 4 , for $\tilde{R}_{0}>1$, we investigate the existence of the endemic equilibrium $\mathbf{E}^{*}$ of system (1.6) for $\tilde{R}_{0}>1$ and the permanence of system (1.6). In Section 5, by means of a direct Lyapunov method, under the condition (1.17), we establish the global asymptotic stability of the endemic equilibrium to complete the proof of Theorem 1.1 and Corollary 1.2.

\section{Positivity and eventual boundedness of solutions}

Adding the first and second equations of (1.6), we have that

$$
\begin{aligned}
\frac{d}{d t}\left\{S_{k}(t)+I_{k}(t)\right\}= & \tilde{b}_{k}(t)-\left(\mu_{k 2}+\tilde{m}_{k k}+m_{k k}\right)\left\{S_{k}(t)+I_{k}(t)\right\} \\
& +\sum_{j=1}^{n} m_{k j} \int_{0}^{h_{2}} g_{k j}(\sigma)\left\{S_{j}(t-\sigma)+I_{j}(t-\sigma)\right\} d \sigma
\end{aligned}
$$

where

$$
\tilde{b}_{k}(t)=b_{k}+\left\{\left(\mu_{k 2}+\gamma_{k}+\tilde{m}_{k k}\right)-\left(\mu_{k 1}+\tilde{l}_{k k}\right)\right\} S_{k}(t)+\sum_{j=1}^{n}\left(l_{k j}-m_{k j}\right) \int_{0}^{h_{2}} g_{k j}(\sigma) S_{j}(t-\sigma) d \sigma,
$$

$k=1,2, \ldots, n$. Thus, for $N_{k}(t)=S_{k}(t)+I_{k}(t), k=1,2, \ldots, n$, we have

$$
\frac{d N_{k}(t)}{d t}=\tilde{b}_{k}(t)-\left(\mu_{k 2}+\tilde{m}_{k k}+m_{k k}\right) N_{k}(t)+\sum_{j=1}^{n} m_{k j} \int_{0}^{h_{2}} g_{k j}(\sigma) N_{j}(t-\sigma) d \sigma
$$

and $\tilde{b}_{k}(t) \leq \overline{\tilde{b}}_{k}, k=1,2, \ldots, n$.

The following lemma shows the positivity and eventual boundedness of $S_{k}$ and $I_{k}, k=$ $1,2, \ldots, n$ of (1.6) (see Muroya et al. [24, Lemma 2.1]).

Lemma 2.1. For system (1.6), it holds that

$$
S_{k}(t)>0, I_{k}(t)>0, \quad \text { for any } k=1,2, \ldots, n \text { and } t>0,
$$

and

$$
\left\{\begin{array}{l}
\limsup _{t \rightarrow+\infty} S_{k}(t) \leq S_{k}^{0}, \quad k=1,2, \ldots, n \\
\limsup _{t \rightarrow+\infty} N_{k}(t) \leq \bar{N}_{k}^{*}, \quad k=1,2, \ldots, n
\end{array}\right.
$$

and $\boldsymbol{\Gamma}$ is a positive invariant set.

Proof. Suppose that there exist a positive constant $t_{1}$ and a positive integer $k_{1} \in\{1,2, \ldots, n\}$ such that $S_{k_{1}}\left(t_{1}\right)=0$ and $S_{k}(t)>0$ for any $0<t<t_{1}$ and $k \in\{1,2, \cdots, n\}$. On the other hand, by (1.6), we have that $S_{k_{1}}^{\prime}\left(t_{1}\right) \geq b_{k_{1}}>0$. This is a contradiction to the fact that 
$S_{k_{1}}(t)>0=S_{k_{1}}\left(t_{1}\right)$ for any $0<t<t_{1}$. Hence, we obtain that $S_{k}(t)>0$ for any $0<t<+\infty$ and $k=1,2, \ldots, n$. By (1.6) and (1.10),

$$
\begin{aligned}
I_{k}(t) & =e^{-\left(\mu_{k 2}+\gamma_{k}+\tilde{m}_{k k}+m_{k k}\right) t} I_{k}(0)+e^{-\left(\mu_{k 2}+\gamma_{k}+\tilde{m}_{k k}+m_{k k}\right) t} \int_{0}^{t} e^{\left(\mu_{k 2}+\gamma_{k}+\tilde{m}_{k k}+m_{k k}\right) u} \\
& \times\left\{S_{k}(u)\left(\sum_{j=1}^{n} \beta_{k j} \int_{0}^{h_{2}} g_{k j}(\sigma) G\left(I_{j}(u-\sigma)\right) d \sigma\right)+\sum_{j=1}^{n}\left(1-\delta_{k j}\right) m_{k j} \int_{0}^{h_{2}} g_{k j}(\sigma) I_{j}(u-\sigma) d \sigma\right\} d u
\end{aligned}
$$

for $k=1,2, \ldots, n$ and $t>0$. This implies that $I_{k}(t)>0$ for any $k=1,2, \ldots, n$ and $t>0$.

On the other hand, for $\bar{S}_{k}:=\limsup _{t \rightarrow+\infty} S_{k}(t), k=1,2, \ldots, n$, by the equation of $S_{k}^{\prime}$, $k=1,2, \ldots, n$ of (1.6), one can see that $\bar{S}_{k}<+\infty, k=1,2, \ldots, n$. Because otherwise, for the positive constants $c_{k}, k=1,2, \ldots, n$ of (1.9), there exists an integer $\bar{k} \in\{1,2, \ldots, n\}$ and a sequence $\left\{t_{p}\right\}_{p=1}^{+\infty}$ such that

$$
\begin{gathered}
\lim _{p \rightarrow+\infty} \frac{S_{\bar{k}}\left(t_{p}\right)}{c_{\bar{k}}}=+\infty, \quad \lim _{p \rightarrow+\infty} \frac{S_{\bar{k}}^{\prime}\left(t_{p}\right)}{c_{\bar{k}}} \geq 0, \quad \text { and } \\
\frac{S_{k}(t)}{c_{k}} \leq \frac{S_{\bar{k}}\left(t_{p}\right)}{c_{\bar{k}}}, \quad \text { for any }-h_{2} \leq t \leq t_{p}, k=1,2 \ldots, n .
\end{gathered}
$$

By the first part of (1.6) and the fluctuation lemma, we have

$$
\begin{aligned}
0 & \leq \frac{S_{\bar{k}}^{\prime}\left(t_{p}\right)}{c_{\bar{k}}} \leq \frac{b_{\bar{k}}}{c_{\bar{k}}}-\mu_{\bar{k} 1} \frac{S_{\bar{k}}\left(t_{p}\right)}{c_{\bar{k}}}+\sum_{j=1}^{n}\left(l_{\bar{k} j} \frac{c_{j}}{c_{\bar{k}}} \int_{0}^{h_{2}} g_{\bar{k} j}(\sigma) \frac{S_{j}(u-\sigma)}{c_{j}} d \sigma-l_{j \bar{k}} \frac{S_{\bar{k}}\left(t_{p}\right)}{c_{\bar{k}}}\right) \\
& \leq \frac{b_{\bar{k}}}{c_{\bar{k}}}-\mu_{\bar{k} 1} \frac{S_{\bar{k}}\left(t_{p}\right)}{c_{\bar{k}}}+\sum_{j=1}^{n} l_{\bar{k} j} \frac{c_{j}}{c_{\bar{k}}} \cdot \frac{S_{\bar{k}}\left(t_{p}\right)}{c_{\bar{k}}}-\left(\sum_{j=1}^{n} l_{j \bar{k}}\right) \frac{S_{\bar{k}}\left(t_{p}\right)}{c_{\bar{k}}} \\
& =\frac{1}{c_{\bar{k}}}\left[b_{\bar{k}}-\left\{c_{\bar{k}} \mu_{\bar{k} 1}+\sum_{j=1}^{n}\left(l_{j \bar{k}} c_{\bar{k}}-l_{\bar{k} j} c_{j}\right)\right\} \frac{S_{\bar{k}}\left(t_{p}\right)}{c_{\bar{k}}}\right],
\end{aligned}
$$

from which we obtain

$$
\frac{S_{\bar{k}}\left(t_{p}\right)}{c_{\bar{k}}} \leq \frac{b_{\bar{k}}}{c_{\bar{k}} \mu_{\bar{k} 1}+\sum_{j=1}^{n}\left(l_{j \bar{k}} c_{\bar{k}}-l_{\bar{k} j} c_{j}\right)}<+\infty, \quad p=1,2, \ldots
$$

This is a contradiction to (2.2).

By (1.12) and the fact that $\mathbf{H}$ defined by (1.11) is an $M$-matrix, we obtain $\bar{S}_{k} \leq S_{k^{\prime}}^{0} k=$ $1,2, \ldots, n$. Thus,

$$
\limsup _{t \rightarrow+\infty} S_{k}(t) \leq S_{k}^{0}, \quad k=1,2, \ldots, n,
$$

which is the first part of (2.1), and $\overline{\tilde{b}}<+\infty, k=1,2, \ldots, n$.

Next, for the solution $\bar{N}_{k}(t)(k=1,2, \ldots, n)$ of the following system:

$$
\frac{d \bar{N}_{k}(t)}{d t}=\overline{\tilde{b}}_{k}-\left(\mu_{k 2}+\tilde{m}_{k k}+m_{k k}\right) \bar{N}_{k}(t)+\sum_{j=1}^{n} m_{k j} \int_{0}^{h_{2}} g_{k j}(\sigma) \bar{N}_{j}(t-\sigma) d \sigma, \quad k=1,2, \ldots, n,
$$

let us consider the following Lyapunov functional:

$$
\left\{\begin{array}{l}
U_{\bar{N}}(t):=\sum_{k=1}^{n}\left\{\bar{N}_{k}^{*} g\left(\frac{\bar{N}_{k}(t)}{\bar{N}_{k}^{*}}\right)+\sum_{j=1}^{n} m_{k j} \bar{N}_{j}^{*} \int_{0}^{h_{2}} g_{k j}(\sigma) \int_{t-\sigma}^{t} g\left(n_{j}(u)\right) d u d \sigma\right\}, \\
g(x):=x-1-\ln x \geq g(1)=0, \text { for } x>0 .
\end{array}\right.
$$


For the usage of the function $g(x)$ in Lyapunov functions, see McCluskey [21] for instance. We show that

$$
\frac{d \bar{U}_{N}(t)}{d t} \leq-\sum_{k=1}^{n}\left\{\mu_{k 2} \bar{N}_{k}^{*} g\left(n_{k}(t)\right)+\overline{\tilde{b}}_{k} g\left(\frac{1}{n_{k}(t)}\right)\right\} \leq 0, n_{k}(t):=\frac{\bar{N}_{k}(t)}{\bar{N}_{k}^{*}}, \quad k=1,2, \ldots, n
$$

and

$$
\lim _{t \rightarrow+\infty} \bar{N}_{k}(t)=\bar{N}_{k}^{*}, \quad k=1,2, \ldots, n
$$

Differentiating $\bar{U}_{N}$ along the solution of (2.3) and using the equilibrium condition $\overline{\tilde{b}}_{k}=$ $\left(\mu_{k 2}+\tilde{m}_{k k}+m_{k k}\right) \bar{N}_{k}^{*}-\sum_{j=1}^{n} m_{k j} \bar{N}_{j}^{*}, k=1,2, \ldots, n$, we obtain

$$
\frac{d \bar{U}_{N}(t)}{d t}=\sum_{k=1}^{n}\left\{\left(1-\frac{\bar{N}_{k}^{*}}{\bar{N}_{k}(t)}\right) \frac{d \bar{N}_{k}(t)}{d t}+\sum_{j=1}^{n} m_{k j} \bar{N}_{j}^{*} \int_{0}^{h_{2}} g_{k j}(\sigma)\left\{g\left(n_{j}(t)\right)-g\left(n_{j}(t-\sigma)\right)\right\} d \sigma\right\}
$$

and

$$
\begin{aligned}
( & \left.1-\frac{\bar{N}_{k}^{*}}{\bar{N}_{k}(t)}\right) \frac{d \bar{N}_{k}(t)}{d t} \\
& =\left(1-\frac{\bar{N}_{k}^{*}}{\bar{N}_{k}(t)}\right)\left\{\tilde{\tilde{b}}_{k}-\left(\mu_{k 2}+\tilde{m}_{k k}+m_{k k}\right) \bar{N}_{k}(t)+\sum_{j=1}^{n} m_{k j} \int_{0}^{h_{2}} g_{k j}(\sigma) \bar{N}_{j}(t-\sigma) d \sigma\right\} \\
& =\left(1-\frac{\bar{N}_{k}^{*}}{\bar{N}_{k}(t)}\right)\left\{-\left(\mu_{k 2}+\tilde{m}_{k k}+m_{k k}\right)\left\{\bar{N}_{k}(t)-\bar{N}_{k}^{*}\right\}+\sum_{j=1}^{n} m_{k j} \int_{0}^{h_{2}} g_{k j}(\sigma)\left\{\bar{N}_{j}(t-\sigma)-N_{j}^{*}\right\} d \sigma\right\} \\
& =\left(1-\frac{1}{n_{k}(t)}\right)\left\{-\left(\mu_{k 2}+\tilde{m}_{k k}+m_{k k}\right) \bar{N}_{k}^{*}\left\{n_{k}(t)-1\right\}+\sum_{j=1}^{n} m_{k j} \bar{N}_{j}^{*} \int_{0}^{h_{2}} g_{k j}(\sigma)\left\{n_{j}(t-\sigma)-1\right\} d \sigma\right\} .
\end{aligned}
$$

It is easy to check that the following equalities hold:

$$
\left(1-\frac{1}{n_{k}(t)}\right)\left\{n_{k}(t)-1\right\}=g\left(n_{k}(t)\right)+g\left(\frac{1}{n_{k}(t)}\right)
$$

and

$$
\left(1-\frac{1}{n_{k}(t)}\right)\left\{n_{j}(t-\sigma)-1\right\}=g\left(n_{j}(t-\sigma)\right)-g\left(\frac{n_{j}(t-\sigma)}{n_{k}(t)}\right)+g\left(\frac{1}{n_{k}(t)}\right) .
$$

It follows that

$$
\begin{aligned}
(1- & \left.\frac{\bar{N}_{k}^{*}}{\bar{N}_{k}(t)}\right) \frac{d \bar{N}_{k}(t)}{d t} \\
= & -\left(\mu_{k 2}+\tilde{m}_{k k}+m_{k k}\right) \bar{N}_{k}^{*}\left\{g\left(n_{k}(t)\right)+g\left(\frac{1}{n_{k}(t)}\right)\right\} \\
& +\sum_{j=1}^{n} m_{k j} \bar{N}_{j}^{*} \int_{0}^{h_{2}} g_{k j}(\sigma)\left\{g\left(n_{j}(t-\sigma)\right)-g\left(\frac{n_{j}(t-\sigma)}{n_{k}(t)}\right)+g\left(\frac{1}{n_{k}(t)}\right)\right\} d \sigma .
\end{aligned}
$$


Thus, we have

$$
\begin{aligned}
\frac{d \bar{U}_{N}(t)}{d t}=\sum_{k=1}^{n}[ & -\left(\mu_{k 2}+\tilde{m}_{k k}+m_{k k}\right) \bar{N}_{k}^{*}\left\{g\left(n_{k}(t)\right)+g\left(\frac{1}{n_{k}(t)}\right)\right\} \\
& +\sum_{j=1}^{n} m_{k j} \bar{N}_{j}^{*} \int_{0}^{h_{2}} g_{k j}(\sigma)\left\{g\left(n_{j}(t-\sigma)\right)-g\left(\frac{n_{j}(t-\sigma)}{n_{k}(t)}\right)+g\left(\frac{1}{n_{k}(t)}\right)\right\} d \sigma \\
& \left.\left.+\sum_{j=1}^{n} m_{k j} \bar{N}_{j}^{*} \int_{0}^{h_{2}} g_{k j}(\sigma)\left\{g\left(n_{j}(t)\right)-g\left(n_{j}(t-\sigma)\right)\right\} d \sigma\right\}\right] \\
=\sum_{k=1}^{n}[ & -\left(\mu_{k 2}+\tilde{m}_{k k}+m_{k k}\right) \bar{N}_{k}^{*}\left\{g\left(n_{k}(t)\right)+g\left(\frac{1}{n_{k}(t)}\right)\right\} \\
& \left.+\sum_{j=1}^{n} m_{k j} \bar{N}_{j}^{*}\left\{g\left(n_{j}(t)\right)-\int_{0}^{h_{2}} g_{k j}(\sigma) g\left(\frac{n_{j}(t-\sigma)}{n_{k}(t)}\right) d \sigma+g\left(\frac{1}{n_{k}(t)}\right)\right\}\right] .
\end{aligned}
$$

Since $\sum_{j=1}^{n} m_{k j} \bar{N}_{j}^{*}=\left(\mu_{k 2}+\tilde{m}_{k k}+m_{k k}\right) \bar{N}_{k}^{*}-\overline{\tilde{b}}_{k}, k=1,2, \ldots, n$, we obtain

$$
\begin{aligned}
& \sum_{k=1}^{n} \sum_{j=1}^{n} m_{k j} \bar{N}_{j}^{*}\left\{g\left(n_{j}(t)\right)+g\left(\frac{1}{n_{k}(t)}\right)\right\} \\
& \quad=\sum_{k=1}^{n}\left(\sum_{j=1}^{n} m_{j k}\right) N_{k}^{*} g\left(n_{k}(t)\right)+\sum_{k=1}^{n}\left(\sum_{j=1}^{n} m_{k j} \bar{N}_{j}^{*}\right) g\left(\frac{1}{n_{k}(t)}\right) \\
& \quad=\sum_{k=1}^{n}\left(\tilde{m}_{k k}+m_{k k}\right) \bar{N}_{k}^{*} g\left(n_{k}(t)\right)+\sum_{k=1}^{n}\left(\sum_{j=1}^{n} m_{k j} \bar{N}_{j}^{*}\right) g\left(\frac{1}{n_{k}(t)}\right) \\
& =\sum_{k=1}^{n}\left(\tilde{m}_{k k}+m_{k k}\right) \bar{N}_{k}^{*} g\left(n_{k}(t)\right)+\sum_{k=1}^{n}\left\{\left(\mu_{k 2}+\tilde{m}_{k k}+m_{k k}\right) \bar{N}_{k}^{*}-\overline{\tilde{b}}_{k}\right\} g\left(\frac{1}{n_{k}(t)}\right) .
\end{aligned}
$$

Hence, we obtain (2.4), which implies (2.5). By the comparison principle, we obtain the second part of (2.1).

By the first and second part of (2.1), it is evident that $\Gamma$ is a positive invariant set. Thus, we obtain the last part of (2.1). This completes the proof.

Lemma 2.2. For any solution of system (1.6) with initial condition (1.7), it holds that

$$
\liminf _{t \rightarrow+\infty} S_{k}(t) \geq \underline{\hat{S}}_{k}:=\frac{b_{k}}{\mu_{k 1}+\tilde{l}_{k k}+\sum_{j=1}^{n} \beta_{k j} G\left(\bar{N}_{j}^{*}\right)}, \quad k=1,2, \ldots, n .
$$

Proof. Let $\left(S_{1}(t), I_{1}(t), R_{1}(t), S_{2}(t), I_{2}(t), R_{2}(t), \ldots, S_{n}(t), I_{n}(t), R_{n}(t)\right)$ be any solution of system (1.6) with initial condition (1.7). By (2.1), it holds that $\limsup _{t \rightarrow+\infty} I_{k}(t) \leq \bar{N}_{k}^{*}, k=$ $1,2, \ldots, n$. This implies that $\varepsilon>0$ sufficiently small, there is a $T_{1}>0$ such that $I_{k}(t)<\bar{N}_{k}^{*}+\varepsilon$ for $t>T_{1}, k=1,2, \ldots, n$. Therefore, from the first part of the hypothesis (1.3) that there exists some sufficiently large positive constant $b$ such that $G(I)$ is monotone increasing on $[0, b]$, we derive

$$
\frac{d S_{k}(t)}{d t} \geq b_{k}-\left\{\mu_{k 1}+\tilde{l}_{k k}+\sum_{j=1}^{n} \beta_{k j} G\left(\bar{N}_{j}^{*}+\varepsilon\right)\right\} S_{k}(t), \quad k=1,2, \ldots, n,
$$

which implies that

$$
\liminf _{t \rightarrow+\infty} S_{k}(t) \geq \frac{b_{k}}{\mu_{k 1}+\tilde{l}_{k k}+\sum_{j=1}^{n} \beta_{k j} G\left(\bar{N}_{j}^{*}+\varepsilon\right)}, \quad k=1,2, \ldots, n .
$$


Since the above inequality holds for arbitrary $\varepsilon>0$ sufficiently small, it immediately follows that

$$
\liminf _{t \rightarrow+\infty} S_{k}(t) \geq \underline{\hat{S}}_{k}, \quad k=1,2, \ldots, n,
$$

which completes the proof.

By Lemma 2.2, for any small positive constant $\varepsilon<\underline{\hat{S}}_{k}$, there exists a positive constant $T_{1}$ such that

$$
S_{k}(t)>\underline{\hat{S}}_{k}-\varepsilon>0, \quad k=1,2, \ldots, n, \quad \text { for any } t \geq T_{1} .
$$

\section{Global stability of the disease-free equilibrium $\mathrm{E}^{0}$ for $\tilde{R}_{0}<1$}

In this section, for $\tilde{R}_{0}<1$, we prove the first part of Theorem 1.1 by means of Lyapunov functional method. We note that there always exists the disease-free equilibrium $\mathbf{E}^{0}=\left(S_{1}^{0}, 0, S_{2}^{0}, 0\right.$, $\left.\ldots, S_{n}^{0}, 0\right)$ of (1.6) in $\Gamma$ such that

$$
b_{k}=\left(\mu_{k 1}+\tilde{l}_{k k}\right) S_{k}^{0}-\sum_{j=1}^{n}\left(1-\delta_{k j}\right) l_{k j} S_{j}^{0}, \quad k=1,2, \ldots, n
$$

holds. We rewrite (1.6) as

$$
\left\{\begin{aligned}
\frac{d S_{k}(t)}{d t}= & b_{k}-\left(\mu_{k 1}+\tilde{l}_{k k}+l_{k k}\right) S_{k}(t) \\
& -\sum_{j=1}^{n}\left(\beta_{k j} S_{k}(t) \int_{0}^{h_{1}} f_{k j}(\tau) G\left(I_{j}(t-\tau)\right) d \tau-l_{k j} \int_{0}^{h_{2}} g_{k j}(\sigma) S_{j}(t-\sigma) d \sigma\right) \\
\frac{d I_{k}(t)}{d t}= & \sum_{j=1}^{n}\left(\beta_{k j} S_{k}(t) \int_{0}^{h_{1}} f_{k j}(\tau) G\left(I_{j}(t-\tau)\right) d \tau+m_{k j} \int_{0}^{h_{2}} g_{k j}(\sigma) I_{j}(t-\sigma) d \sigma\right) \\
& -\left(\mu_{k 2}+\gamma_{k}+\tilde{m}_{k k}+m_{k k}\right) I_{k}(t), \quad k=1,2 \ldots, n .
\end{aligned}\right.
$$

Proof of the first part of Theorem 1.1 for $\tilde{\boldsymbol{R}}_{\mathbf{0}}<\mathbf{1}$. It is sufficient to show that the disease-free equilibrium $\mathbf{E}^{0}=\left(S_{1}^{0}, 0, S_{2}^{0}, 0, \ldots, S_{n}^{0}, 0\right)$ of $(1.6)$ is globally asymptotically stable.

Assume that (1.16) holds and for this positive vector $\left(u_{1}, u_{2}, \ldots, u_{n}\right)$, consider the following Lyapunov functional:

$$
\begin{aligned}
W(t)=\sum_{k=1}^{n} u_{k}[ & S_{k}^{0} g\left(\frac{S_{k}(t)}{S_{k}^{0}}\right)+I_{k}(t)+\sum_{j=1}^{n}\left\{\beta_{k j} S_{k}^{0} \int_{0}^{h_{1}} f_{k j}(\tau) \int_{t-\tau}^{t} G\left(I_{j}(u)\right) d u d \tau\right. \\
& \left.\left.\quad+l_{k j} S_{j}^{0} \int_{0}^{h_{2}} g_{k j}(\sigma) \int_{t-\sigma}^{t} g\left(\frac{S_{j}(u)}{S_{j}^{0}}\right) d u d \sigma+m_{k j} \int_{0}^{h_{2}} g_{k j}(\sigma) \int_{t-\sigma}^{t} I_{j}(u) d u d \sigma\right\}\right], \\
g(x)=x-1- & \ln x \geq g(1)=0, \quad \text { for any } x>0 .
\end{aligned}
$$


Differentiating $W(t)$ along the solution of (1.6), we have

$$
\begin{aligned}
& \frac{d W(t)}{d t}=\sum_{k=1}^{n} u_{k}\left\{\left(1-\frac{S_{k}^{0}}{S_{k}(t)}\right) \frac{d S_{k}(t)}{d t}+\frac{d I_{k}(t)}{d t}\right\} \\
&+\sum_{k=1}^{n} u_{k} \sum_{j=1}^{n}\left[\beta_{k j} S_{k}^{0} \int_{0}^{h_{1}} f_{k j}(\tau)\left\{G\left(I_{j}(t)\right)-G\left(I_{j}(t-\tau)\right)\right\} d \tau\right. \\
&+l_{k j} S_{j}^{0} \int_{0}^{h_{2}} g_{k j}(\sigma)\left\{g\left(\frac{S_{j}(t)}{S_{j}^{0}}\right)-g\left(\frac{S_{j}(t-\sigma)}{S_{j}^{0}}\right)\right\} d \sigma \\
&\left.+m_{k j} \int_{0}^{h_{2}} g_{k j}(\sigma)\left\{I_{j}(t)-I_{j}(t-\sigma)\right\} d \sigma\right] .
\end{aligned}
$$

Put

$$
x_{k}^{0}(t)=\frac{S_{k}(t)}{S_{k}^{0}}, \quad k=1,2, \ldots, n .
$$

By (3.1) and (3.2), we have that

$$
\begin{aligned}
\frac{d S_{k}(t)}{d t}= & b_{k}-\left(\mu_{k 1}+\tilde{l}_{k k}+l_{k k}\right) S_{k}(t) \\
& -\sum_{j=1}^{n}\left(\beta_{k j} S_{k}(t) \int_{0}^{h_{1}} f_{k j}(\tau) G\left(I_{j}(t-\tau)\right) d \tau-l_{k j} \int_{0}^{h_{2}} g_{k j}(\sigma) S_{j}(t-\sigma) d \sigma\right) \\
= & -\left(\mu_{k 1}+\tilde{l}_{k k}+l_{k k}\right)\left(S_{k}(t)-S_{k}^{0}\right) \\
& -\sum_{j=1}^{n}\left(\beta_{k j} S_{k}(t) \int_{0}^{h_{1}} f_{k j}(\tau) G\left(I_{j}(t-\tau)\right) d \tau-l_{k j} \int_{0}^{h_{2}} g_{k j}(\sigma)\left(S_{j}(t-\sigma)-S_{j}^{0}\right) d \sigma\right),
\end{aligned}
$$

and

$$
\begin{aligned}
\frac{d I_{k}(t)}{d t}= & \sum_{j=1}^{n}\left(\beta_{k j} S_{k}(t) \int_{0}^{h_{1}} f_{k j}(\tau) G\left(I_{j}(t-\tau)\right) d \tau+m_{k j} \int_{0}^{h_{2}} g_{k j}(\sigma) I_{j}(t-\sigma) d \sigma\right) \\
& -\left(\mu_{k 2}+\gamma_{k}+\tilde{m}_{k k}+m_{k k}\right) I_{k}(t) .
\end{aligned}
$$

Then,

$$
\begin{aligned}
& \frac{d W(t)}{d t}=\sum_{k=1}^{n} u_{k}\left[( 1 - \frac { S _ { k } ^ { 0 } } { S _ { k } ( t ) } ) \left\{-\left(\mu_{k 1}+\tilde{l}_{k k}+l_{k k}\right)\left(S_{k}(t)-S_{k}^{0}\right)\right.\right. \\
& -\sum_{j=1}^{n}\left(\beta_{k j} S_{k}(t) \int_{0}^{h_{1}} f_{k j}(\tau) G\left(I_{j}(t-\tau)\right) d \tau\right. \\
& \left.\left.\quad-l_{k j} \int_{0}^{h_{2}} g_{k j}(\sigma)\left(S_{j}(t-\sigma)-S_{j}^{0}\right) d \sigma\right)\right\} \\
& +\left\{\sum_{j=1}^{n}\left(\beta_{k j} S_{k}(t) \int_{0}^{h_{1}} f_{k j}(\tau) G\left(I_{j}(t-\tau)\right) d \tau+m_{k j} \int_{0}^{h_{2}} g_{k j}(\sigma) I_{j}(t-\sigma) d \sigma\right)\right. \\
& \left.-\left(\mu_{k 2}+\gamma_{k}+\tilde{m}_{k k}+m_{k k}\right) I_{k}(t)\right\} \\
& +\sum_{j=1}^{n}\left(\beta_{k j} S_{k}^{0} \int_{0}^{h_{1}} f_{k j}(\tau)\left\{G\left(I_{j}(t)\right)-G\left(I_{j}(t-\tau)\right)\right\} d \tau\right.
\end{aligned}
$$




$$
\begin{aligned}
& +l_{k j} S_{j}^{0} \int_{0}^{h_{2}} g_{k j}(\sigma)\left\{g\left(x_{j}^{0}(t)\right)-g\left(x_{j}^{0}(t-\sigma)\right)\right\} d \sigma \\
& \left.\left.+m_{k j} \int_{0}^{h_{2}} g_{k j}(\sigma)\left\{I_{j}(t)-I_{j}(t-\sigma)\right\} d \sigma\right)\right] \\
& =\sum_{k=1}^{n} u_{k}\left[( 1 - \frac { S _ { k } ^ { 0 } } { S _ { k } ( t ) } ) \left\{-\left(\mu_{k 1}+\tilde{l}_{k k}+l_{k k}\right) S_{k}^{0}\left(\frac{S_{k}(t)}{S_{k}^{0}}-1\right)\right.\right. \\
& \left.+\sum_{j=1}^{n} l_{k j} S_{j}^{0} \int_{0}^{h_{2}} g_{k j}(\sigma)\left(\frac{S_{j}(t-\sigma)}{S_{j}^{0}}-1\right) d \sigma\right\} \\
& -\sum_{j=1}^{n}\left(\beta_{k j} S_{k}(t) \int_{0}^{h_{1}} f_{k j}(\tau) G\left(I_{j}(t-\tau)\right) d \tau-\beta_{k j} S_{k}^{0} \int_{0}^{h_{1}} f_{k j}(\tau) G\left(I_{j}(t-\tau)\right) d \tau\right) \\
& +\left\{\sum_{j=1}^{n}\left(\beta_{k j} S_{k}(t) \int_{0}^{h_{1}} f_{k j}(\tau) G\left(I_{j}(t-\tau)\right) d \tau+m_{k j} \int_{0}^{h_{2}} g_{k j}(\sigma) I_{j}(t-\sigma) d \sigma\right)\right. \\
& \left.-\left(\mu_{k 2}+\gamma_{k}+\tilde{m}_{k k}+m_{k k}\right) I_{k}(t)\right\} \\
& +\sum_{j=1}^{n}\left(\beta_{k j} S_{k}^{0} \int_{0}^{h_{1}} f_{k j}(\tau)\left\{G\left(I_{j}(t)\right)-G\left(I_{j}(t-\tau)\right)\right\} d \tau\right. \\
& +l_{k j} S_{j}^{0} \int_{0}^{h_{2}} g_{k j}(\sigma)\left\{g\left(x_{j}^{0}(t)\right)-g\left(x_{j}^{0}(t-\sigma)\right)\right\} d \sigma \\
& \left.\left.+m_{k j} \int_{0}^{h_{2}} g_{k j}(\sigma)\left\{I_{j}(t)-I_{j}(t-\sigma)\right\} d \sigma\right)\right] \\
& =-\sum_{k=1}^{n} u_{k}\left[\left(\mu_{k 1}+\tilde{l}_{k k}+l_{k k}\right) S_{k}^{0}\left(1-\frac{1}{x_{k}^{0}(t)}\right)\left(x_{k}^{0}(t)-1\right)\right. \\
& \left.-\sum_{j=1}^{n} l_{k j} S_{j}^{0} \int_{0}^{h_{2}} g_{k j}(\sigma)\left(1-\frac{1}{x_{k}^{0}(t)}\right)\left(x_{j}^{0}(t-\sigma)-1\right) d \sigma\right] \\
& +\sum_{k=1}^{n} u_{k}\left\{\sum_{j=1}^{n}\left(\beta_{k j} S_{k}^{0} G\left(I_{j}(t)\right)+\left(1-\delta_{k j}\right) m_{k j} I_{j}(t)\right)-\left(\mu_{k 2}+\gamma_{k}+\tilde{m}_{k k}\right) I_{k}(t)\right\} \\
& +\sum_{k=1}^{n} u_{k} \sum_{j=1}^{n} l_{k j} S_{j}^{0} \int_{0}^{h_{2}} g_{k j}(\sigma)\left\{g\left(x_{j}^{0}(t)\right)-g\left(x_{j}^{0}(t-\sigma)\right)\right\} d \sigma .
\end{aligned}
$$

Let us now consider the first part of the last equation in (3.3). Since

$$
\left(1-\frac{1}{x_{k}^{0}(t)}\right)\left(x_{k}^{0}(t)-1\right)=x_{k}^{0}(t)+\frac{1}{x_{k}^{0}(t)}-2=g\left(x_{k}^{0}(t)\right)+g\left(\frac{1}{x_{k}^{0}(t)}\right)
$$

and

$$
\begin{aligned}
\left(1-\frac{1}{x_{k}^{0}(t)}\right)\left(x_{j}^{0}(t-\sigma)-1\right) & =x_{j}^{0}(t-\sigma)-\frac{x_{j}^{0}(t-\sigma)}{x_{k}^{0}(t)}+\frac{1}{x_{j}^{0}(t)}-1 \\
& =g\left(x_{j}^{0}(t-\sigma)\right)-g\left(\frac{x_{j}^{0}(t-\sigma)}{x_{k}^{0}(t)}\right)+g\left(\frac{1}{x_{k}^{0}(t)}\right),
\end{aligned}
$$


and by (1.10), it holds that

$$
\begin{aligned}
\sum_{k=1}^{n} u_{k}\left(\mu_{k 1}+\tilde{l}_{k k}+l_{k k}\right) S_{k}^{0}\left(1-\frac{1}{x_{k}^{0}(t)}\right)\left(x_{k}^{0}(t)-1\right) \\
=\sum_{k=1}^{n} u_{k}\left(\mu_{k 1}+\tilde{l}_{k k}+l_{k k}\right) S_{k}^{0}\left\{g\left(x_{k}^{0}(t)\right)+g\left(\frac{1}{x_{k}^{0}(t)}\right)\right\}
\end{aligned}
$$

and

$$
\begin{aligned}
\sum_{k=1}^{n} u_{k} & \sum_{j=1}^{n} l_{k j} S_{j}^{0} \int_{0}^{h_{2}} g_{k j}(\sigma)\left(1-\frac{1}{x_{k}^{0}(t)}\right)\left(x_{j}^{0}(t-\sigma)-1\right) d \sigma \\
\quad= & \sum_{k=1}^{n} u_{k} \sum_{j=1}^{n} l_{k j} S_{j}^{0} \int_{0}^{h_{2}} g_{k j}(\sigma)\left\{g\left(x_{j}^{0}(t-\sigma)\right)-g\left(\frac{x_{j}^{0}(t-\sigma)}{x_{k}^{0}(t)}\right)+g\left(\frac{1}{x_{k}^{0}(t)}\right)\right\} d \sigma .
\end{aligned}
$$

It follows from (3.3), (3.4) and (3.5) that

$$
\begin{aligned}
& \frac{d W(t)}{d t}=-\sum_{k=1}^{n} u_{k}\left(\mu_{k 1}+\tilde{l}_{k k}+l_{k k}\right) S_{k}^{0}\left\{g\left(x_{k}^{0}(t)\right)+g\left(\frac{1}{x_{k}^{0}(t)}\right)\right\} \\
& +\sum_{k=1}^{n} u_{k} \sum_{j=1}^{n} l_{k j} S_{j}^{0} \int_{0}^{h_{2}} g_{k j}(\sigma)\left\{g\left(x_{j}^{0}(t-\sigma)\right)-g\left(\frac{x_{j}^{0}(t-\sigma)}{x_{k}^{0}(t)}\right)+g\left(\frac{1}{x_{k}^{0}(t)}\right)\right\} d \sigma \\
& +\sum_{k=1}^{n} u_{k}\left\{\sum_{j=1}^{n}\left(\beta_{k j} S_{k}^{0} G\left(I_{j}(t)\right)+\left(1-\delta_{k j}\right) m_{k j} I_{j}(t)\right)-\left(\mu_{k 2}+\gamma_{k}+\tilde{m}_{k k}\right) I_{k}(t)\right\} \\
& +\sum_{k=1}^{n} u_{k} \sum_{j=1}^{n} l_{k j} S_{j}^{0} \int_{0}^{h_{2}} g_{k j}(\sigma)\left\{g\left(x_{j}^{0}(t)\right)-g\left(x_{j}^{0}(t-\sigma)\right)\right\} d \sigma \\
& =-\sum_{k=1}^{n}\left(u_{k}\left(\mu_{k 1}+\tilde{l}_{k k}\right)-\sum_{j=1}^{n} u_{j}\left(1-\delta_{j k}\right) l_{j k}\right) S_{k}^{0} g\left(x_{k}^{0}(t)\right) \\
& -\sum_{k=1}^{n} u_{k}\left\{\left(\mu_{k 1}+\tilde{l}_{k k}\right) S_{k}^{0}-\sum_{j=1}^{n}\left(1-\delta_{k j}\right) l_{k j} S_{j}^{0}\right\} g\left(\frac{1}{x_{k}^{0}(t)}\right) \\
& -\sum_{k=1}^{n} u_{k} \sum_{j=1}^{n}\left(1-\delta_{k j}\right) l_{k j} S_{j}^{0} g\left(\frac{x_{j}^{0}(t-\sigma)}{x_{k}^{0}(t)}\right) \\
& +\sum_{k=1}^{n} u_{k}\left\{\sum_{j=1}^{n}\left(\beta_{k j} S_{k}^{0}+\left(1-\delta_{k j}\right) m_{k j}\right) I_{j}(t)-\left(\mu_{k 2}+\gamma_{k}+\tilde{m}_{k k}\right) I_{k}(t)\right\} \\
& +\sum_{k=1}^{n} u_{k} \sum_{j=1}^{n} \beta_{k j} S_{k}^{0}\left\{G\left(I_{j}(t)\right)-I_{j}(t)\right\} \\
& =-\sum_{k=1}^{n}\left(u_{k}\left(\mu_{k 1}+\tilde{l}_{k k}\right)-\sum_{j=1}^{n} u_{j}\left(1-\delta_{j k}\right) l_{j k}\right) S_{k}^{0} g\left(x_{k}^{0}(t)\right)-\sum_{k=1}^{n} u_{k} b_{k} g\left(\frac{1}{x_{k}^{0}(t)}\right) \\
& -\sum_{k=1}^{n} u_{k} \sum_{j=1}^{n}\left(1-\delta_{k j}\right) l_{k j} S_{j}^{0} g\left(\frac{x_{j}^{0}(t-\sigma)}{x_{k}^{0}(t)}\right) \\
& +\sum_{k=1}^{n}\left\{\sum_{j=1}^{n} u_{j}\left(\beta_{j k} S_{j}^{0}+\left(1-\delta_{j k}\right) m_{j k}\right)-u_{k}\left(\mu_{k 2}+\gamma_{k}+\tilde{m}_{k k}\right)\right\} I_{k}(t) \\
& +\sum_{k=1}^{n} u_{k} \sum_{j=1}^{n} \beta_{k j} S_{k}^{0}\left\{G\left(I_{j}(t)\right)-I_{j}(t)\right\} \leq 0 .
\end{aligned}
$$


Let us note that we make use of assumption (1.2), (1.16) and $G\left(I_{j}(t)\right) \leq I_{j}(t)$.

It is obvious from (3.6) that $\mathcal{R}_{0}<1$ if and only if $S_{k}(t)=S_{k}^{0}$ and $I_{k}(t) \equiv 0, k=1,2, \ldots, n$. Thus, it follows from the classical LaSalle's invariance principle (see [13]) that the disease-free equilibrium $\mathbf{E}^{0}$ of the system (1.6) is globally asymptotically stable. Hence, we obtain the first part $\tilde{R}_{0}<1$ of Theorem 1.1.

It should also be noted that if $\mathcal{R}_{0}=1$, then from (3.6), we can not show $S_{k}(t)=S_{k}^{0}$ and $I_{k}(t) \equiv 0, k=1,2, \ldots, n$.

\section{Existence of the endemic equilibrium and permanence for $\tilde{R}_{0}>1$}

In this section, our attention is focused on the case $\tilde{R}_{0}>1$. First, we show the existence of an endemic equilibrium $\mathbf{E}^{*}$ and the second, we show the permanence of system (1.6). Finally, we prove the global asymptotic stability of it by applying Lyapunov functional method.

By (1.13), it follows from the Perron-Frobenius theorem that $\tilde{R}_{0}=\rho\left(\tilde{\mathbf{M}}\left(\mathbf{S}^{0}\right)\right)$ is an eigenvalue of matrix $\tilde{\mathbf{M}}\left(\mathbf{S}^{0}\right)$ associated with a positive (right) eigenvector $\omega=\left(\omega_{1}, \omega_{2}, \ldots, \omega_{n}\right)$ such that $\omega_{k}>0, k=1,2, \ldots, n$. For such $\omega$, we have

$$
\tilde{\mathbf{M}}\left(\mathbf{S}^{0}\right) \omega=\rho\left(\tilde{\mathbf{M}}\left(\mathbf{S}^{0}\right)\right) \omega,
$$

which is equivalent to

$$
\sum_{j=1}^{n}\left\{S_{k}^{0} \beta_{k j}+\left(1-\delta_{k j}\right) m_{k j}\right\} \omega_{j}-\rho\left(\tilde{\mathbf{M}}\left(\mathbf{S}^{0}\right)\right)\left(\mu_{k 2}+\gamma_{k}+m_{k k}\right) \omega_{k}=0 .
$$

Thus, by $\rho\left(\tilde{\mathbf{M}}\left(\mathbf{S}^{0}\right)\right)=\tilde{R}_{0}>1$, it holds that

$$
\sum_{j=1}^{n}\left\{\beta_{k j} S_{k}^{0}+\left(1-\delta_{k j}\right) m_{k j}\right\} \omega_{j}-\left(\mu_{k 2}+\gamma_{k}+\tilde{m}_{k k}\right) \omega_{k}>0, \quad k=1,2, \ldots, n .
$$

\subsection{Existence of the endemic equilibrium}

First, we prove the existence of an endemic equilibrium $\mathbf{E}^{*}$ of system (1.6) when $\tilde{R}_{0}>1$.

Lemma 4.1. Let $\tilde{R}_{0}$ and $\Gamma$ be defined by (1.13) and (1.14), respectively. If $\tilde{R}_{0}>1$, then system (1.6) has an endemic equilibrium $\mathbf{E}^{*}=\left(S_{1}^{*}, I_{1}^{*}, S_{2}^{*}, I_{2}^{*}, \ldots, S_{n}^{*}, I_{n}^{*}\right) \in \Gamma^{0}$ such that

$$
\left\{\begin{array}{l}
b_{k}=\left(\mu_{k 1}+\tilde{l}_{k k}\right) S_{k}^{*}+\sum_{j=1}^{n} \beta_{k j} S_{k}^{*} G\left(I_{j}^{*}\right)-\sum_{j=1}^{n}\left(1-\delta_{k j}\right) l_{k j} S_{j}^{*}, \\
\left(\mu_{k 2}+\gamma_{k}+\tilde{m}_{k k}\right) I_{k}^{*}=\sum_{j=1}^{n} \beta_{k j} S_{k}^{*} G\left(I_{j}^{*}\right)+\sum_{j=1}^{n}\left(1-\delta_{k j}\right) m_{k j} I_{j}^{*}, \quad k=1,2, \ldots, n .
\end{array}\right.
$$

Proof. Since $\tilde{R}_{0}>1$ implies that for $\rho\left(\tilde{\mathbf{M}}\left(\mathbf{S}^{0}\right)\right)>1$, there exists a positive eigenvector $\omega=$ $\left(\omega_{1}, \omega_{2}, \ldots, \omega_{n}\right)^{T}$ such that $\tilde{\mathbf{M}}\left(\mathbf{S}^{0}\right) \omega=\rho\left(\tilde{\mathbf{M}}\left(\mathbf{S}^{0}\right)\right) \omega$, where $S_{k}^{0} \leq \bar{N}_{k}^{*}, k=1,2, \ldots, n$ and $\tilde{F}\left(\mathbf{S}^{0}\right)=\left[S_{k}^{0} \beta_{k j}+\left(1-\delta_{k j}\right) m_{k j}\right]_{n \times n}$ and $\tilde{V}=\left[\delta_{k j}\left(\mu_{k 2}+\gamma_{k}+\tilde{m}_{k k}\right)\right]_{n \times n}$ are $n \times n$ matrices.

Since by Lemma 2.1, $S_{k}^{*}+I_{k}^{*}=N_{k}^{*}=S_{k}^{0}, k=1,2, \ldots, n$ if the endemic equilibrium exists, we may consider the existence of solution $\mathbf{x}=\left(x_{1}, x_{2}, \ldots, x_{n}\right)$ of the system $\mathbf{F}(\mathbf{x})=\mathbf{0}$, where

$$
\left\{\begin{array}{l}
\mathbf{F}(\mathbf{x})=\left(f_{1}(\mathbf{x}), f_{2}(\mathbf{x}), \ldots, f_{n}(\mathbf{x})\right)^{T}=\left[f_{k}(\mathbf{x})\right]_{n \times 1}, \quad \mathbf{x}=\left(x_{1}, x_{2}, \ldots, x_{n}\right)^{T}, \\
f_{k}(\mathbf{x})=-\left(s_{k}(\mathbf{x}) \sum_{j=1}^{n} \beta_{k j} G\left(x_{j}\right)-\left(\mu_{k 2}+\gamma_{k}+\tilde{m}_{k k}\right) x_{k}+\sum_{j=1}^{n}\left(1-\delta_{k j}\right) m_{k j} x_{j}\right)
\end{array}\right.
$$


under the conditions $0<x_{k}<\bar{N}_{k}^{*}$ and

$$
\left(\sum_{j=1}^{n} \beta_{k j} G\left(x_{j}\right)+\left(\mu_{k 1}+\tilde{l}_{k k}\right)\right) S_{k}(\mathbf{x})-\sum_{j=1}^{n}\left(1-\delta_{k j}\right) l_{k j} S_{j}(\mathbf{x})-b_{k}=0
$$

for $k=1,2, \ldots, n$. Let us also note that $x_{k}(k=1,2, \ldots, n)$ correspond to the number of infected individual for patch $k(k=1,2, \ldots, n)$ appeared in the endemic equilibrium $\mathbf{E}^{*}$. One can see that

$$
\begin{aligned}
\mathbf{F}(\mathbf{x}) & =-\left[S_{k}(\mathbf{x}) \sum_{j=1}^{n} \beta_{k j} G\left(x_{j}\right)-\left(\mu_{k 2}+\gamma_{k}+\tilde{m}_{k k}\right) x_{k}+\sum_{j=1}^{n}\left(1-\delta_{k j}\right) m_{k j} x_{j}\right]_{n \times 1} \\
& =-\left(\tilde{F}\left(\mathbf{S}^{0}\right) \mathbf{x}-\tilde{V} \mathbf{x}\right)-\left[S_{k}(\mathbf{x}) \beta_{k j} \frac{G\left(x_{j}\right)}{x_{j}}-S_{k}^{0} \beta_{k j}\right]_{n \times n} \mathbf{x}
\end{aligned}
$$

and hence,

$$
\left\{\begin{array}{l}
\mathbf{F}(\omega)=-\left(\tilde{F}\left(\mathbf{S}^{0}\right) \omega-\tilde{V} \omega\right)-\left[S_{k}(\omega) \beta_{k j} \frac{G\left(\omega_{j}\right)}{\omega_{j}}-S_{k}^{0} \beta_{k j}\right]_{n \times n} \omega, \quad \text { and } \\
-\left(\tilde{F}\left(\mathbf{S}^{0}\right) \omega-\tilde{V} \omega\right)=-\tilde{V}\left\{\tilde{\mathbf{M}}\left(\mathbf{S}^{0}\right) \omega-\omega\right\}=-\tilde{V}\left\{\rho\left(\tilde{\mathbf{M}}\left(\mathbf{S}^{0}\right)\right)-1\right\} \omega<\mathbf{0},
\end{array}\right.
$$

where the ordering of vectors in $\mathbb{R}^{n}$ is the usual element-wise one in $\mathbb{R}^{n}$.

For any $\alpha>0$, we have

$$
\mathbf{F}(\alpha \omega)=\alpha\left\{-\left(\tilde{F}\left(\mathbf{S}^{0}\right) \omega-\tilde{V} \omega\right)-\left[S_{k}(\alpha \omega) \beta_{k j} \frac{G\left(\alpha \omega_{j}\right)}{\alpha \omega_{j}}-S_{k}^{0} \beta_{k j}\right]_{n \times n} \omega\right\},
$$

and by assumption (1.3) and (2.1), $\lim _{I \rightarrow+0} I / G(I)=1$ and by (1.12) and the last part of (4.1), one can see that $\lim _{\alpha \rightarrow 0} S_{k}(\alpha \omega)=S_{k}^{0}, k=1,2, \ldots, n$. Thus, there exists a small positive constant $\underline{\alpha}<1$ such that

$$
\mathbf{F}(\underline{\alpha} \omega) \leq \mathbf{0} .
$$

On the other hand, from the second and the last part of (4.1), we have

$$
\left(\mu_{k 1}+\tilde{l}_{k k}\right) S_{k}(\mathbf{x})-\sum_{j=1}^{n}\left(1-\delta_{k j}\right) l_{k j} S_{j}(\mathbf{x})=b_{k}+\sum_{j=1}^{n}\left(1-\delta_{k j}\right) m_{k j} x_{j}-\left(\mu_{k 2}+\gamma_{k}+\tilde{m}_{k k}\right) x_{k} .
$$

Let $A=\left[a_{k j}\right]_{n \times n}$ be a $n \times n$ matrix defined by

$$
a_{k k}=\mu_{k 1}+\tilde{l}_{k k}, \quad a_{k j}=-l_{k j}, \quad j \neq k, k=1,2, \ldots, n .
$$

Then, by the theory of M-matrices, $A=\left[a_{k j}\right]_{n \times n}$ is a nonsingular M-matrix and there exists its nonnegative positive inverse matrix $A^{(-1)}=\left[a_{k j}^{(-1)}\right]_{n \times n}, a_{k j}>0, k, j=1,2, \ldots, n$ and from (4.2),

$$
\begin{aligned}
S_{k}(\mathbf{x}) & =\sum_{j=1}^{n} a_{k j}^{(-1)}\left(b_{j}+\sum_{l=1}^{n}\left(1-\delta_{j l}\right) m_{j l} x_{l}-\left(\mu_{j 2}+\gamma_{j}+\tilde{m}_{j j}\right) x_{j}\right) \\
& \leq\left(\max _{k, j=1,2, \ldots, n} a_{k j}^{(-1)}\right) \sum_{j=1}^{n}\left(b_{j}+\sum_{l=1}^{n}\left(1-\delta_{j l}\right) m_{j l} x_{l}-\left(\mu_{j 2}+\gamma_{j}+\tilde{m}_{j j}\right) x_{j}\right) \\
& =\left(\max _{k, j=1,2, \ldots, n} a_{k j}^{(-1)}\right) \sum_{j=1}^{n}\left(b_{j}-\left(\mu_{j 2}+\gamma_{j}\right) x_{j}\right),
\end{aligned}
$$


where we use the relation $\sum_{j=1}^{n} \sum_{l=1}^{n}\left(1-\delta_{j l}\right) m_{j l} x_{l}=\sum_{j=1}^{n} \sum_{l=1}^{n}\left(1-\delta_{j l}\right) m_{l j} x_{j}=\sum_{j=1}^{n} \tilde{m}_{j j} x_{j}$. Therefore, for $\overline{\mathbf{x}}=(\bar{x}, \bar{x}, \bar{x}, \ldots, \bar{x})$ with $\bar{x}>0$, we have

$$
\begin{aligned}
f_{k}(\overline{\mathbf{x}})=- & \left(S_{k}(\overline{\mathbf{x}}) \sum_{j=1}^{n} \beta_{k j} G(\bar{x})-\left(\mu_{k 2}+\gamma_{k}+\tilde{m}_{k k}\right) \bar{x}+\sum_{j=1}^{n}\left(1-\delta_{k j}\right) m_{k j} \bar{x}\right) \\
\geq- & \left\{\left(\max _{k, j=1,2, \ldots, n} a_{k j}^{(-1)}\right) \sum_{j=1}^{n}\left(b_{j}-\left(\mu_{j 2}+\gamma_{j}\right) \bar{x}\right)\right\} \sum_{j=1}^{n} \beta_{k j} \bar{x} \\
& \left.-\left(\mu_{k 2}+\gamma_{k}+\tilde{m}_{k k}\right) \bar{x}+\sum_{j=1}^{n}\left(1-\delta_{k j}\right) m_{k j} \bar{x}\right] \\
\geq- & {\left[\left\{\max _{k, j=1,2, \ldots, n} a_{k j}^{(-1)}\right) \sum_{j=1}^{n}\left(b_{j}-\left(\mu_{j 2}+\gamma_{j}\right) \bar{x}\right)\right\} \sum_{j=1}^{n} \beta_{k j} } \\
& \left.-\left(\mu_{k 2}+\gamma_{k}+\tilde{m}_{k k}\right)+\sum_{j=1}^{n}\left(1-\delta_{k j}\right) m_{k j}\right] \bar{x},
\end{aligned}
$$

and hence, for a sufficiently large positive constant $\bar{x}$, we obtain $\mathbf{F}(\overline{\mathbf{x}}) \geq \mathbf{0}$.

Moreover, for system (4.1), the elements of the Fréchet derivative $\mathbf{F}^{\prime}(\mathbf{x})=\left[\partial f_{k} / \partial x_{j}\right]$ are

$$
\begin{aligned}
& \frac{\partial f_{k}\left(x_{1}, x_{2}, \ldots, x_{n}\right)}{\partial x_{j}} \\
& =\left\{\begin{array}{l}
-\frac{\partial S_{k}(\overline{\mathbf{x}})}{\partial x_{k}} \sum_{j=1}^{n} \beta_{k j} G\left(x_{j}\right)-S_{k}(\overline{\mathbf{x}}) \beta_{k k} G^{\prime}\left(x_{k}\right)+\left(\mu_{k 2}+\gamma_{k}+\tilde{m}_{k k}\right), \text { for } j=k, k=1,2, \ldots, n, \\
-\frac{\partial S_{k}(\overline{\mathbf{x}})}{\partial x_{j}} \sum_{j=1}^{n} \beta_{k j} G\left(x_{j}\right)-S_{k}(\overline{\mathbf{x}}) \beta_{k j} G^{\prime}\left(x_{j}\right)-m_{k j} \leq 0, \text { for } j \neq k, k=1,2, \ldots, n,
\end{array}\right.
\end{aligned}
$$

where by the last part of $(4.1), \frac{\partial S_{k}(\overline{\mathbf{x}})}{\partial x_{l}}, l=1,2, \ldots, n$ satisfy

$$
\left(\sum_{j=1}^{n} \beta_{k j} G\left(x_{j}\right)+\left(\mu_{k 1}+\tilde{l}_{k k}\right)\right) \frac{\partial S_{k}(\overline{\mathbf{x}})}{\partial x_{l}}-\sum_{j=1}^{n}\left(1-\delta_{k j}\right) l_{k j} \frac{\partial S_{k}(\overline{\mathbf{x}})}{\partial x_{l}}=-\beta_{k j} G^{\prime}\left(x_{j}\right), \quad k=1,2, \ldots, n,
$$

from which by the monotone property on the argument of function $G$ and the theory of Mmatrices (see, for example, Berman and Plemmons [3] or Varga [31]), one can easily see that

$$
\frac{\partial S_{k}(\overline{\mathbf{x}})}{\partial x_{l}} \leq 0, \quad j \neq k, \quad k=1,2, \ldots, n .
$$

Then, for a sufficiently large positive constant $\bar{l}>0$ such that

$$
\begin{gathered}
\bar{l}>\max \left\{\max _{1 \leq k \leq n}\left(-\frac{\partial S_{k}(\overline{\mathbf{x}})}{\partial x_{k}} \sum_{j=1}^{n} \beta_{k j} G\left(x_{j}\right)-S_{k}(\overline{\mathbf{x}}) \beta_{k k} G^{\prime}\left(x_{k}\right)+\left(\mu_{k 2}+\gamma_{k}+\tilde{m}_{k k}\right)\right),\right. \\
\left.\frac{\partial S_{k}(\overline{\mathbf{x}})}{\partial x_{j}} \sum_{j=1}^{n} \beta_{k j} G\left(x_{j}\right)+S_{k}(\overline{\mathbf{x}}) \beta_{k j} G^{\prime}\left(x_{j}\right)+m_{k j}\right\},
\end{gathered}
$$

$B=\left[\delta_{k j} \bar{l}^{-1}\right]$ becomes a non-negative non-singular matrix and subinverse (see Ortega and Rheinboldt [27] or Muroya [23]) of $\mathbf{F}^{\prime}(\mathbf{x})$, i.e., $B \mathbf{F}^{\prime}(\mathbf{x}) \leq I$ and $\mathbf{F}^{\prime}(\mathbf{x}) B \leq I$ for the unit matrix $I$ and any $\mathbf{x}$ with $0<x_{k}<\bar{x}_{k}, k=1,2, \ldots, n$. Thus, to the equation $\mathbf{F}(\mathbf{x})=\mathbf{0}$ in $\mathbb{R}^{n}$, we can apply the monotone iteration such that $\mathbf{x}_{0}=\underline{\alpha} \omega, \mathbf{y}_{0}=\overline{\mathbf{x}}$ with $\mathbf{F}\left(\mathbf{x}_{0}\right) \leq \mathbf{0} \leq \mathbf{F}\left(\mathbf{y}_{0}\right)$ 
and $\mathbf{x}_{p+1}=\mathbf{x}_{p}-B \mathbf{F}\left(\mathbf{x}_{p}\right), p=1,2, \ldots, n$ of Ortega and Rheinboldt [27, Theorem 4.1 and Corollary 4.1] or Muroya [23, Theorem 3.1], we have a monotone increasing vector sequence $\mathbf{x}_{0} \leq \mathbf{x}_{1} \leq \ldots \leq \mathbf{x}_{p} \leq \mathbf{x}_{p+1} \leq \mathbf{y}_{0}$ and a $\operatorname{limit}_{\lim } \rightarrow+\infty \mathbf{x}_{p}=\mathbf{x}^{*}=\left(x_{1}^{*}, x_{2}^{*}, \ldots, x_{n}^{*}\right)$ which is a solution of $\mathbf{F}(\mathbf{x})=\mathbf{0}$. Hence, we conclude that there exists an endemic equilibrium of (1.6).

\subsection{Permanence}

Now, we show the permanence of system (1.6). Let $\underline{I}:=\min _{1 \leq j \leq n} \lim \inf _{t \rightarrow+\infty} I_{j}(t) / I_{j}^{*}$. Without loss of generality, we can assume that $k \in\{1,2, \ldots, n\}$ is an integer such that $\liminf _{t \rightarrow+\infty} I_{k}(t) / I_{k}^{*} \equiv \underline{I}$. Let us set

$$
\mathbf{j}_{k}=\left\{j \in\{1,2, \ldots, n\} \mid \beta_{k j}>0\right\}
$$

By (1.8), we have $\mathbf{j}_{k} \neq \varnothing$.

Extending the result of Enatsu et al. [6, Lemma 4.1] on permanence to the model (1.6), we introduce the following lemmas, which play a key role to obtain the permanence.

Lemma 4.2. For $R_{0}>1$, if

$$
\left\{\begin{array}{l}
S_{k}(t) \geq S_{k}^{*}, \quad \frac{G\left(I_{j}(t-\tau)\right)}{G\left(I_{j}^{*}\right)} \geq \frac{I_{k}(t)}{I_{k}^{*}}, \quad \text { for any } \quad 0 \leq \tau \leq h_{1}, \quad \text { and } \\
\frac{I_{j}(t)}{I_{j}^{*}} \geq \frac{I_{k}(t-\sigma)}{I_{k}^{*}}, \quad \text { for any } \quad 0 \leq \sigma \leq h_{2}, \quad j=1,2, \ldots, n,
\end{array}\right.
$$

then $I_{k}^{\prime}(t) \geq 0$. If $I_{k}^{\prime}(t) \leq 0$, then

$$
\left\{\begin{array}{l}
S_{k}(t) \leq S_{k^{\prime}}^{*} \quad \text { or } \quad \frac{G\left(I_{j}(t-\tau)\right)}{G\left(I_{j}^{*}\right)} \leq \frac{I_{k}(t)}{I_{k}^{*}}, \quad \text { for any } 0 \leq \tau \leq h_{1}, \quad \text { or } \\
\frac{I_{j}(t-\sigma)}{I_{j}^{*}} \leq \frac{I_{k}(t-\sigma)}{I_{k}^{*}}, \quad \text { for any } \quad 0 \leq \sigma \leq h_{2}, \quad j=1,2, \ldots, n .
\end{array}\right.
$$

Proof. Assume that $R_{0}>1$. By the second equations of (1.6) and (4.1), we have that

$$
\begin{aligned}
I_{k}^{\prime}(t)= & \sum_{j=1}^{n} \beta_{k j} S_{k}(t) G\left(I_{j}^{*}\right) \int_{0}^{h_{1}} f_{k j}(\tau) \frac{G\left(I_{j}(t-\tau)\right)}{G\left(I_{j}^{*}\right)} d \tau-\left(\mu_{k 2}+\gamma_{k}+\tilde{m}_{k k}+m_{k k}\right) I_{k}^{*} \frac{I_{k}(t)}{I_{k}^{*}} \\
& +\sum_{j=1}^{n} m_{k j} I_{j}^{*} \int_{0}^{h_{2}} g_{k j}(\sigma) \frac{I_{j}(t-\sigma)}{I_{j}^{*}} d \sigma \\
= & \sum_{j=1}^{n} \beta_{k j} S_{k}(t) G\left(I_{j}^{*}\right) \int_{0}^{h_{1}} f_{k j}(\tau) \frac{G\left(I_{j}(t-\tau)\right)}{G\left(I_{j}^{*}\right)} d \tau-\left(\sum_{j=1}^{n} \beta_{k j} S_{k}^{*} G\left(I_{j}^{*}\right)+\sum_{j=1}^{n} m_{k j} I_{j}^{*}\right) \frac{I_{k}(t)}{I_{k}^{*}} \\
& +\sum_{j=1}^{n} m_{k j} I_{j}^{*} \int_{0}^{h_{2}} g_{k j}(\sigma) \frac{I_{j}(t-\sigma)}{I_{j}^{*}} d \sigma \\
= & \sum_{j=1}^{n} \beta_{k j}\left\{S_{k}(t)-S_{k}^{*}\right\} \int_{0}^{h_{1}} f_{k j}(\tau) G\left(I_{j}(t-\tau)\right) d \tau \\
& +\sum_{j=1}^{n} \beta_{k j} S_{k}^{*} G\left(I_{j}^{*}\right) \int_{0}^{h_{1}} f_{k j}(\tau)\left(\frac{G\left(I_{j}(t-\tau)\right)}{G\left(I_{j}^{*}\right)}-\frac{I_{k}(t)}{I_{k}^{*}}\right) d \tau \\
& +\sum_{j=1}^{n} m_{k j} I_{j}^{*} \int_{0}^{h_{2}} g_{k j}(\sigma)\left(\frac{I_{j}(t-\sigma)}{I_{j}^{*}}-\frac{I_{k}(t)}{I_{k}^{*}}\right) d \sigma
\end{aligned}
$$

from which we obtain the conclusion of this lemma. 
For a positive constant $T_{1}$, the following lemma holds true.

Lemma 4.3. Assume that $0<q<1$ and there exist $a t \geq T_{1}$ and some $k \in\{1,2, \ldots, n\}$ such that $I_{k}^{\prime}(t) \leq 0$. If there exists an integer $\bar{j} \in \mathbf{j}_{k}$ such that

$$
\frac{I_{\bar{j}}(t-\tau)}{I_{\vec{j}}^{*}}>q, \quad \text { for any } \quad 0 \leq \tau \leq h_{1},
$$

then

$$
I_{k}(t)>\underline{I}_{k}(\bar{j} ; q):=\frac{\left(\underline{\hat{S}}_{k}-\varepsilon\right) \beta_{k j} G\left(q I_{\dot{j}}^{*}\right)}{\mu_{k 2}+\gamma_{k}+\tilde{m}_{k k}+m_{k k}}>0 .
$$

Proof. Assume that $0<q<1$. If there exist a $t \geq T_{1}$, some $k \in\{1,2, \ldots, n\}$ and an integer $\bar{j} \in \mathbf{j}_{k}$ such that (4.3) holds. Then, by (2.6) and (1.8), $S_{k}(t)>\underline{\hat{S}}_{k}-\varepsilon>0$ and $\beta_{k \bar{j}}>0$, and by the second equation of (1.6),

$$
\begin{aligned}
0 \geq I_{k}^{\prime}(t)= & S_{k}(t) \sum_{j=1}^{n} \beta_{k j} \int_{0}^{h_{1}} f_{k j}(\tau) G\left(I_{j}(t-\tau)\right) d \tau-\left(\mu_{k 2}+\gamma_{k}+\tilde{m}_{k k}+m_{k k}\right) I_{k}(t) \\
& +\sum_{j=1}^{n} m_{k j} \int_{0}^{h_{2}} g_{k j}(\sigma) I_{j}(t-\sigma) d \sigma \\
> & \left(\underline{\hat{S}}_{k}-\varepsilon\right) \beta_{k j} G\left(q I_{j}^{*}\right)-\left(\mu_{k 2}+\gamma_{k}+\tilde{m}_{k k}+m_{k k}\right) I_{k}(t),
\end{aligned}
$$

from which we have

$$
I_{k}(t)>\frac{\left(\underline{\hat{S}}_{k}-\varepsilon\right) \beta_{k j} G\left(q I_{\dot{j}}^{*}\right)}{\mu_{k 2}+\gamma_{k}+\tilde{m}_{k k}+m_{k k}}>0 .
$$

Thus, (4.4) holds. This completes the proof.

Lemma 4.4. If $R_{0}>1$, then for any solution of system (1.6) with initial condition (1.7), it holds that

$$
\liminf _{t \rightarrow+\infty} I_{k}(t) \geq \underline{\hat{I}}_{q}:=\underline{\hat{I}}(q) \exp \left\{-\max _{1 \leq k \leq n}\left(\mu_{k 2}+\gamma_{k}+\tilde{m}_{k k}+m_{k k}\right) \rho(q)\right\}, \quad k=1,2, \ldots, n,
$$

where for $0<q<1$, put

$$
\left\{\begin{array}{l}
\underline{\underline{I}}(q):=\min _{1 \leq k \leq n, \bar{j} \in \mathbf{j}_{k}} \underline{I}_{k}(\bar{j} ; q)>0, \\
q_{G}=\max _{1 \leq j \leq n} \frac{G\left(q I_{j}^{*}\right)}{G\left(I_{j}^{*}\right)}<1, \quad \text { and } r_{k}=\mu_{k 1}+\tilde{l}_{k k}+l_{k k}+q_{G} \sum_{j=1}^{n} \beta_{k j} G\left(I_{j}^{*}\right),
\end{array}\right.
$$

and $q$ is chosen sufficiently small and $\rho(q)>0$ is chosen sufficiently large to satisfy the following inequalities:

$$
S_{k}^{*}<\frac{b_{k}}{r_{k}}, \quad \text { and } \quad S_{k}^{*}<S_{k}^{\triangle}:=\frac{b_{k}}{r_{k}}\left(1-e^{-r_{k} \rho(q)}\right), \quad k=1,2, \ldots, n .
$$

Proof. Assume that $\tilde{R}_{0}>1$ and let $\left(S_{1}(t), I_{1}(t), S_{2}(t), I_{2}(t), \ldots, S_{n}(t), I_{n}(t)\right)$ be any solution of system (1.6) with initial condition (1.7), and $0<q<1$ and $\rho(q)>0$ are a fixed sufficiently small constant and a large constant, respectively, such that (4.6) holds.

For $t \geq T_{1}$, let $k(t) \in\{1,2, \ldots, n\}$ be defined by

$$
\frac{I_{k(t)}(t)}{I_{k(t)}^{*}}=\min _{1 \leq j \leq n} \frac{I_{j}(t)}{I_{j}^{*}}, \quad \text { for any } t \geq T_{1},
$$


and put

$$
\underline{I}(t):=I_{k(t)}(t), \underline{S}(t)=S_{k(t)}(t) \text {, and } \underline{\beta}_{j}=\beta_{k(t) j}, \text { for any } j \in \mathbf{j}_{k(t)} \text {, and } t \geq T_{1} .
$$

Hereafter, for simplicity without loss of generality and for the readers' convenience, it is better to express $k(t)$ temporally as $k$ for each $t \geq T_{1}$.

Put

$$
D_{k}(q)=\left\{t \geq T_{1} \mid \frac{I_{j}(t-\tau)}{I_{j}^{*}} \leq q, \text { for any } j \in \mathbf{j}_{k} \text {, and } 0 \leq \tau \leq h_{1}\right\}, \quad k=1,2, \ldots, n,
$$

and for $t \geq T_{1}$,

$$
\underline{I}(t)=\frac{I_{k}(t)}{I_{k}^{*}},
$$

where $k=k(t) \in\{1,2, \ldots, n\}$ is an integer such that (4.7) holds.

Because by Lemma 4.3, for $t \geq T_{1}$,

$$
I_{k}(t)>\underline{I}_{k}(q):=\min _{\bar{j} \in \mathbf{j}_{k}} \underline{I}_{k}(\bar{j} ; q)>0, \quad \text { if } t \notin D_{k}(q),
$$

to prove that there exists a positive constant $\underline{\underline{I}}$ independent from the initial conditions (1.7) such that

$$
\underline{I}=\min _{1 \leq j \leq n} \liminf _{t \rightarrow+\infty} \frac{I_{j}(t)}{I_{j}^{*}} \geq \underline{\hat{I}},
$$

we may hereafter restrict our attention to the case that there exists a sufficiently large $t \in$ $D_{k}(q)$.

We now prove that it is impossible that there exists a nonnegative constant $t_{0} \geq T_{1}$ such that $t \in D_{q}(k)$ for all $t \geq t_{0}$. Suppose on the contrary that $t \in D_{q}(k)$ for all $t \geq t_{0}$. Consider the following functional:

$V(t)=I_{k}(t)+\sum_{j=1}^{n}\left(\beta_{k j} \int_{0}^{h_{1}} f_{k j}(\tau) \int_{t-\tau}^{t} S_{k}(u+\tau) G\left(I_{j}(u)\right) d u d \tau+m_{k j} \int_{0}^{h_{2}} g_{k j}(\sigma) \int_{t-\sigma}^{t} I_{j}(u) d u d \sigma\right)$.

By (4.5) and the inequality $G\left(I_{j}(t-\tau)\right) \leq G\left(q I_{j}^{*}\right) \leq q_{G} G\left(I_{j}^{*}\right), j=1,2, \ldots, n$ for $t \geq t_{0}+h_{1}$, one can obtain that

$$
\frac{d S_{k}(t)}{d t} \geq b_{k}-\left(\mu_{k}+\tilde{l}_{k k}+l_{k k}+q_{G} \sum_{j=1}^{n} \beta_{k j} G\left(I_{j}^{*}\right)\right) S_{k}(t)=b_{k}-r_{k} S_{k}(t), \text { for } t \geq t_{0}+h_{1},
$$

which yields

$$
\begin{aligned}
S_{k}(t) & \geq e^{-r_{k}\left(t-t_{0}\right)}\left(S_{k}\left(t_{0}\right)+b_{k} \int_{t_{0}}^{t} e^{r_{k}\left(s-t_{0}\right)} d s\right) \\
& >\frac{b_{k}}{r_{k}}\left(1-e^{-r_{k}\left(t-t_{0}\right)}\right), \quad \text { for } t \geq t_{0}+h_{1} .
\end{aligned}
$$

Hence, if we choose $\rho(q)>0$ sufficiently large to satisfy $S_{k}^{*}<S_{k}^{\triangle}:=b_{k}\left(1-e^{-r_{k} \rho(q)}\right) / r_{k}$ of (4.6), then it follows from (4.9) that

$$
S_{k}(t)>\frac{b_{k}}{r_{k}}\left(1-e^{-r_{k} \rho(q)}\right)=S_{k}^{\triangle}>S_{k}^{*}, \text { for } t \geq t_{0}+h_{1}+\rho(q) .
$$


Since $t \in D_{k}(q)$ implies $I_{j}(t-\tau) / I_{j}^{*} \leq q<1$ for $j \in \mathbf{j}_{k}, 0 \leq \tau \leq h_{1}$ and $t \geq T_{1}$, we have $I_{j}(t) \leq I_{j}^{*}$ for $j \in \mathbf{j}_{k}$ and $t \geq t_{0}+h_{1}$. By the second part of the hypothesis (1.3), it holds that $G\left(I_{j}(t)\right) / I_{j}(t) \geq G\left(I_{j}^{*}\right) / I_{j}^{*}$, which is equivalent to $I_{j}(t) / I_{j}^{*} \leq G\left(I_{j}(t)\right) / G\left(I_{j}^{*}\right), j \in \mathbf{j}_{k}$ for $t \geq t_{0}+h_{1}$. Thus, by $I_{k}(t) / I_{k}^{*} \leq I_{j}(t) / I_{j}^{*}, j=1,2, \ldots, n$, we obtain $I_{k}(t) / I_{k}^{*} \leq G\left(I_{j}(t)\right) / G\left(I_{j}^{*}\right)$, $j=1,2, \ldots, n$ for $t \geq t_{0}+h_{1}$.

By the above discussion and Lemma 4.2, we obtain $I_{k}^{\prime}(t) \geq 0$ for any $t \geq t_{0}+h_{1}+\rho(q)$, and there exists a $j \in\{1,2, \ldots, n\}$ and an $s_{t} \in\left[t-h_{1}, t\right)$ such that $I_{k}(t) \geq I_{j}\left(s_{t}\right)$. For a positive constant $\hat{I} \equiv \min _{t_{0}+\rho(q) \leq s \leq t_{0}+h_{1}+\rho(q)} I_{j}(s)>0$, we thus obtain that

$$
I_{k}(t) \geq \hat{I} \quad \text { for any } t \geq t_{0}+h_{1}+\rho(q) .
$$

Calculating the derivative of $V(t)$ along solutions of system (1.6) gives as follows.

$$
\begin{aligned}
\frac{d V(t)}{d t}= & \sum_{j=1}^{n} \beta_{k j} S_{k}(t) \int_{0}^{h_{1}} f_{k j}(\tau) G\left(I_{j}(t-\tau)\right) d \tau-\left(\mu_{k 2}+\gamma_{k}+\tilde{m}_{k k}+m_{k k}\right) I_{k}(t) \\
& +\sum_{j=1}^{n} m_{k j} \int_{0}^{h_{2}} g_{k j}(\sigma) I_{j}(t-\sigma) d \sigma \\
& +\sum_{j=1}^{n}\left(\beta_{k j} \int_{0}^{h_{1}} f_{k j}(\tau)\left\{S_{k}(t+\tau) G\left(I_{j}(t)\right)-S_{k}(t) G\left(I_{j}(t-\tau)\right)\right\} d \tau\right. \\
& \left.\quad+m_{k j} \int_{0}^{h_{2}} g_{k j}(\sigma)\left\{I_{j}(t)-I_{j}(t-\sigma)\right\} d \sigma\right) \\
= & \sum_{j=1}^{n} \beta_{k j} \int_{0}^{h_{1}} f_{k j}(\tau) S_{k}(t+\tau) G\left(I_{j}(t)\right) d \tau-\left(\mu_{k 2}+\gamma_{k}+\tilde{m}_{k k}+m_{k k}\right) I_{k}^{*} \frac{I_{k}(t)}{I_{k}^{*}}+\sum_{j=1}^{n} m_{k j} J_{j}^{*} \frac{I_{j}(t)}{I_{j}^{*}} \\
\geq & \sum_{j=1}^{n} \beta_{k j} S_{k}^{\triangle} G\left(I_{j}^{*}\right) \frac{G\left(I_{j}(t)\right)}{G\left(I_{j}^{*}\right)}-\left(\mu_{k 2}+\gamma_{k}+\tilde{m}_{k k}+m_{k k}\right) I_{k}^{*} \frac{I_{k}(t)}{I_{k}^{*}}+\sum_{j=1}^{n} m_{k j} I_{j}^{*} \frac{I_{j}(t)}{I_{j}^{*}} \\
\geq & \sum_{j=1}^{n} \beta_{k j} S_{k}^{\triangle} G\left(I_{j}^{*}\right) \frac{I_{j}(t)}{I_{j}^{*}}-\sum_{j=1}^{n} \beta_{k j} S_{k}^{*} G\left(I_{j}^{*}\right) \frac{I_{k}(t)}{I_{k}^{*}}+\sum_{j=1}^{n} m_{k j} I_{j}^{*}\left(\frac{I_{j}(t)}{I_{j}^{*}}-\frac{I_{k}(t)}{I_{k}^{*}}\right) \\
\geq & \left(S_{k}^{\triangle}-S_{k}^{*}\right) \sum_{j=1}^{n} \beta_{k j} G\left(I_{j}^{*}\right) \frac{I_{k}(t)}{I_{k}^{*}}, \quad \text { for } t \geq t_{0}+h_{1}+\rho(q),
\end{aligned}
$$

because by (4.10), $S_{k}(t) \geq S_{k}^{\triangle}>S_{k}^{*}$ for $t \geq t_{0}+h_{1}+\rho(q)$ and by $t \in D_{k}(q)$ and the definition of $k$ and the above note, we have that $G\left(I_{j}(t)\right) / G\left(I_{j}^{*}\right) \geq I_{j}(t) / I_{j}^{*} \geq I_{k}(t) / I_{k}^{*}$ for $t \geq t_{0}+h_{1}$.

Thus, $\lim _{t \rightarrow+\infty} V(t)=+\infty$. However, from (2.1) and the first part of the hypothesis (1.3), it holds that

$$
\limsup _{t \rightarrow+\infty} V(t) \leq \bar{N}_{k}^{*}+\sum_{j=1}^{n}\left\{\beta_{k j} S_{k}^{0} h_{1} G\left(\bar{N}_{j}^{*}\right)+m_{k j} h_{2} \bar{N}_{j}^{*}<+\infty\right.
$$

This leads to a contradiction. Hence the claim is proved.

By the claim and (4.8), there exist sufficiently large constants $t_{1}<t_{2}$ and $t_{1}, t_{2} \in D_{k}(q)$ such that for any $t_{1}<t<t_{2}, t \in D_{k}(q)$, but there exists a $0 \leq t_{3}<t_{1}$ and $t_{4}>t_{2}$ such that $t_{3}, t_{4} \notin D_{k}(q)$.

We now show that $I(t) \geq \underline{\underline{I}}_{q}$ for all $t$ sufficiently large $t \in D_{k}(q)$. Let $t_{1}<t_{2}$ be sufficiently large constants $t_{1}, t_{2} \in D_{k}(q)$ such that

$$
I_{k}\left(t_{1}\right) \geq \underline{I}_{k}(q), I_{k}\left(t_{2}\right) \geq \underline{I}_{k}(q), t \in D_{k}(q), \quad \text { for any } t_{1}<t<t_{2} .
$$


Since from the second equation of system (1.6), we have

$$
\frac{d I_{k}(t)}{d t} \geq-\left(\mu_{k 2}+\gamma_{k}+\tilde{m}_{k k}+m_{k k}\right) I_{k}(t), \quad \text { for } t \geq t_{1}
$$

Then,

$$
\begin{aligned}
I_{k}(t) & \geq I_{k}\left(t_{1}\right) e^{-\left(\mu_{k 1}+\gamma_{k}+\tilde{m}_{k k}+m_{k k}\right)\left(t-t_{1}\right)} \\
& \geq \hat{I}_{k}(q) e^{-\left(\mu_{k 2}+\gamma_{k}+\tilde{m}_{k k}+m_{k k}\right) \rho(q)} \\
& \geq \hat{I}_{q}, \quad \text { for } t_{1} \leq t \leq t_{1}+\rho(q) .
\end{aligned}
$$

If $t_{2}>t_{1}+\rho(q)$, then by applying the similar discussion to (4.10) and (4.11) in place of $t_{0}$ by $t_{1}$, we obtain $I_{k}(t) \geq \underline{I}_{q}$ for $t_{1}+\rho(q)<t \leq t_{2}$. Hence, we prove that $I(t) \geq \underline{I}_{q}$ for $t_{1} \leq t \leq t_{2}$.

Since the interval $t_{1} \leq t \leq t_{2}$ in $D_{k}(q)$ is arbitrarily chosen, we can easily conclude that $I(t) \geq \underline{\underline{I}}_{q}$ holds for all $t$ sufficiently large $t \in D_{k}(q)$. Thus, we obtain that $\lim _{\inf } \hat{t}_{t \rightarrow+\infty} I(t) \geq \underline{\underline{I}}_{q}$. This completes the proof.

By (2.1) and Lemmas 2.2, 4.2-4.4, we obtain the permanence of system (1.6) for $R_{0}>1$.

\section{Global stability of the endemic equilibrium $\mathrm{E}^{*}$ for $\tilde{R}_{0}>1$}

In this section, we assume $\tilde{R}_{0}>1$. By Lemma 4.1 , there exists an endemic equilibrium

$$
\mathbf{E}^{*}=\left(S_{1}^{*}, I_{1}^{*}, S_{2}^{*}, I_{2}^{*}, \ldots, S_{n}^{*}, I_{n}^{*}\right)
$$

of (1.6) in $\Gamma^{0}$ such that (4.1) holds. We rewrite (1.6) as

$$
\left\{\begin{aligned}
\frac{d S_{k}(t)}{d t}= & b_{k}-\left(\mu_{k 1}+\tilde{l}_{k k}+l_{k k}\right) S_{k}(t) \\
& -\sum_{j=1}^{n}\left(\beta_{k j} S_{k}(t) \int_{0}^{h_{1}} f_{k j}(\tau) G\left(I_{j}(t-\tau)\right) d \tau-l_{k j} \int_{0}^{h_{2}} g_{k j}(\sigma) S_{j}(t-\sigma) d \sigma\right), \\
\frac{d I_{k}(t)}{d t}= & \sum_{j=1}^{n}\left(\beta_{k j} S_{k}(t) \int_{0}^{h_{1}} f_{k j}(\tau) G\left(I_{j}(t-\tau)\right) d \tau+m_{k j} \int_{0}^{h_{2}} g_{k j}(\sigma) I_{j}(t-\sigma) d \sigma\right) \\
& -\left(\mu_{k 2}+\gamma_{k}+\tilde{m}_{k k}+m_{k k}\right) I_{k}(t), \quad k=1,2 \ldots, n .
\end{aligned}\right.
$$

Let us set

$$
\begin{aligned}
U(t):=\sum_{k=1}^{n} v_{k}[ & S_{k}^{*} g\left(\frac{S_{k}(t)}{S_{k}^{*}}\right)+I_{k}^{*} g\left(\frac{I_{k}(t)}{I_{k}^{*}}\right) \\
+\sum_{j=1}^{n}\{ & \beta_{k j} S_{k}^{*} G\left(I_{j}^{*}\right) \int_{0}^{h_{1}} f_{k j}(\tau) \int_{t-\tau}^{t} g\left(\frac{G\left(I_{j}(u)\right)}{G_{j}\left(I_{j}^{*}\right)}\right) d u d \tau \\
& +l_{k j} S_{j}^{*} \int_{0}^{h_{2}} g_{k j}(\sigma) \int_{t-\sigma}^{t} g\left(\frac{S_{j}(u)}{S_{j}^{*}}\right) d u d \sigma \\
& \left.\left.+m_{k j} I_{j}^{*} \int_{0}^{h_{2}} g_{k j}(\sigma) \int_{t-\sigma}^{t} g\left(\frac{I_{j}(u)}{I_{j}^{*}}\right) d u d \sigma\right\}\right],
\end{aligned}
$$


where $v_{1}, v_{2}, \ldots, v_{n}$ will be appropriately chosen later (see (1.17)). Differentiating $U$ along the solutions of (1.6), we have

$$
\begin{aligned}
& \frac{d U(t)}{d t}=\sum_{k=1}^{n} v_{k}\{(\left.\left(1-\frac{S_{k}^{*}}{S_{k}(t)}\right) \frac{d S_{k}(t)}{d t}+\left(1-\frac{I_{k}^{*}}{I_{k}(t)}\right) \frac{d I_{k}(t)}{d t}\right\} \\
&+\sum_{k=1}^{n} v_{k}\left[\sum_{j=1}^{n} \beta_{k j} S_{k}^{*} G\left(I_{j}^{*}\right) \int_{0}^{h_{1}} f_{k j}(\tau)\left\{g\left(\frac{G\left(I_{j}(t)\right)}{G\left(I_{j}^{*}\right)}\right)-g\left(\frac{G\left(I_{j}(t-\tau)\right)}{G\left(I_{j}^{*}\right)}\right)\right\} d \tau\right. \\
&+\sum_{j=1}^{n} l_{k j} S_{j}^{*} \int_{0}^{h_{2}} g_{k j}(\sigma)\left\{g\left(\frac{S_{j}(t)}{S_{j}^{*}}\right)-g\left(\frac{S_{j}(t-\sigma)}{S_{j}^{*}}\right)\right\} d \sigma \\
&\left.+\sum_{j=1}^{n} m_{k j} I_{j}^{*} \int_{0}^{h_{2}} g_{k j}(\sigma)\left\{g\left(\frac{I_{j}(t)}{I_{j}^{*}}\right)-g\left(\frac{I_{j}(t-\sigma)}{I_{j}^{*}}\right)\right\} d \sigma\right]
\end{aligned}
$$

For simplicity, we put

$$
x_{k}(t)=\frac{S_{k}(t)}{S_{k}^{*}}, \quad y_{k}(t)=\frac{I_{k}(t)}{I_{k}^{*}}, \quad \tilde{y}_{j}(t)=\frac{G\left(I_{j}(t)\right)}{G\left(I_{j}^{*}\right)}, \quad k, j=1,2, \ldots, n .
$$

From (4.1) and (5.1), we derive that for $k=1,2, \ldots, n$,

$$
\begin{aligned}
\frac{d S_{k}(t)}{d t}= & b_{k}-\left(\mu_{k 1}+\tilde{l}_{k k}+l_{k k}\right) S_{k}(t) \\
& -\sum_{j=1}^{n}\left(\beta_{k j} S_{k}(t) \int_{0}^{h_{1}} f_{k j}(\tau) G\left(I_{j}(t-\tau)\right) d \tau-l_{k j} \int_{0}^{h_{2}} g_{k j}(\sigma) S_{j}(t-\sigma) d \sigma\right) \\
= & -\left(\mu_{k 1}+\tilde{l}_{k k}+\tilde{l}_{k k}+l_{k k}\right)\left(S_{k}(t)-S_{k}^{*}\right) \\
& -\sum_{j=1}^{n}\left\{\beta_{k j}\left(S_{k}(t) \int_{0}^{h_{1}} f_{k j}(\tau) G\left(I_{j}(t-\tau)\right) d \tau-S_{k}^{*} G\left(I_{j}^{*}\right)\right)\right. \\
& \left.\quad-l_{k j} \int_{0}^{h_{2}} g_{k j}(\sigma)\left(S_{j}(t-\sigma)-S_{j}^{*}\right) d \sigma\right\} \\
= & -\left(\mu_{k 1}+\tilde{l}_{k k}+l_{k k}\right) S_{k}^{*}\left(x_{k}(t)-1\right) \\
& -\sum_{j=1}^{n}\left(\beta_{k j} S_{k}^{*} G\left(I_{j}^{*}\right) \int_{0}^{h_{1}} f_{k j}(\tau)\left(x_{k}(t) \tilde{y}_{j}(t-\tau)-1\right) d \tau\right. \\
& \left.\quad-l_{k j} S_{j}^{*} \int_{0}^{h_{2}} g_{k j}(\sigma)\left(x_{j}(t-\sigma)-1\right) d \sigma\right),
\end{aligned}
$$

and

$$
\begin{aligned}
\frac{d I_{k}(t)}{d t}= & \sum_{j=1}^{n}\left(\beta_{k j} S_{k}(t) \int_{0}^{h_{1}} f_{k j}(\tau) G\left(I_{j}(t-\tau)\right) d \tau+m_{k j} \int_{0}^{h_{2}} g_{k j}(\sigma) I_{j}(t-\sigma)\right) \\
& -\left(\mu_{k 2}+\gamma_{k}+\tilde{m}_{k k}+m_{k k}\right) I_{k}(t) \\
= & \sum_{j=1}^{n}\left(\beta_{k j} S_{k}^{*} G\left(I_{j}^{*}\right) \int_{0}^{h_{1}} f_{k j}(\tau) x_{k}(t) \tilde{y}_{j}(t-\tau) d \tau+m_{k j} I_{j}^{*} \int_{0}^{h_{2}} g_{k j}(\sigma) y_{j}(t-\sigma) d \sigma\right) \\
& -\left(\mu_{k 2}+\gamma_{k}+\tilde{m}_{k k}+m_{k k}\right) I_{k}^{*} y_{k}(t) \\
= & \sum_{j=1}^{n}\left(\beta_{k j} S_{k}^{*} G\left(I_{j}^{*}\right) \int_{0}^{h_{1}} f_{k j}(\tau)\left(x_{k}(t) \tilde{y}_{j}(t-\tau)-y_{k}(t)\right) d \tau\right. \\
& \left.\quad+m_{k j} I_{j}^{*} \int_{0}^{h_{2}} g_{k j}(\sigma)\left(y_{j}(t-\sigma)-y_{k}(t)\right) d \sigma\right) .
\end{aligned}
$$


Then, we have

$$
\begin{aligned}
& \sum_{k=1}^{n} v_{k}\left\{\left(1-\frac{S_{k}^{*}}{S_{k}(t)}\right) \frac{d S_{k}(t)}{d t}+\right. \\
& =\sum_{k=1}^{n} v_{k}\left[\left(1-\frac{1}{x_{k}(t)}\right)\left\{-\left(I_{k}^{*}\right) \frac{d I_{k}(t)}{d t}\right\}\right. \\
& -\sum_{j=1}^{n}\left(\tilde{l}_{k k}+l_{k k}\right) S_{k}^{*}\left(x_{k}(t)-1\right) \\
& \left.\left.\quad-l_{k j} S_{j}^{*} \int_{0}^{h_{2}} g_{k j}^{*}(\sigma)\left(x_{j}(t-\sigma)-1\right) d \sigma\right)\right\} \\
& +\left(1-\frac{1}{y_{k}(t)}\right) \sum_{j=1}^{n}\left(\beta_{k j} S_{k}^{*} G\left(I_{j}^{*}\right) \int_{0}^{h_{1}} f_{k j}(\tau)\left(x_{k}(t) \tilde{y}_{j}(t-\tau)-y_{k}(t)\right) d \tau\right. \\
& \left.\left.+m_{k j} I_{j}^{*} \int_{0}^{h_{2}} g_{k j}(\sigma)\left(y_{j}(t-\sigma)-y_{k}(t)\right) d \sigma\right)\right] \\
& =-\sum_{k=1}^{n} v_{k}\left[\left(\mu_{k 1}+\tilde{l}_{k k}+l_{k k}\right) S_{k}^{*}\left(1-\frac{1}{x_{k}(t)}\right)\left(x_{k}(t)-1\right)\right. \\
& \left.\quad-\sum_{j=1}^{n} l_{k j} S_{j}^{*} \int_{0}^{h_{2}} g_{k j}(\sigma)\left(1-\frac{1}{x_{k}(t)}\right)\left(x_{j}(t-\sigma)-1\right) d \sigma\right] \\
& +\sum_{k=1}^{n} v_{k}\left[\sum _ { j = 1 } ^ { n } \beta _ { k j } S _ { k } ^ { * } G ( I _ { j } ^ { * } ) \int _ { 0 } ^ { h _ { 1 } } f _ { k j } ( \tau ) \left\{\left(1-\frac{1}{x_{k}(t)}\right)\left(1-x_{k}(t) \tilde{y}_{j}(t-\tau)\right)\right.\right. \\
& \left.+\left(1-\frac{1}{y_{k}(t)}\right)\left(x_{k}(t) \tilde{y}_{j}(t-\tau)-y_{k}(t)\right)\right\} d \tau
\end{aligned}
$$

For the first part of the last equation in (5.3), we have

$$
\begin{aligned}
\left(1-\frac{1}{x_{k}(t)}\right)\left(x_{k}(t)-1\right) & =x_{k}(t)+\frac{1}{x_{k}(t)}-2 \\
& =g\left(x_{k}(t)\right)+g\left(\frac{1}{x_{k}(t)}\right)
\end{aligned}
$$

and

$$
\begin{aligned}
\left(1-\frac{1}{x_{k}(t)}\right)\left(x_{j}(t-\sigma)-1\right) & =x_{j}(t-\sigma)-\frac{x_{j}(t-\sigma)}{x_{k}(t)}+\frac{1}{x_{k}(t)}-1 \\
& =g\left(x_{j}(t-\sigma)\right)-g\left(\frac{x_{j}(t-\sigma)}{x_{k}(t)}\right)+g\left(\frac{1}{x_{k}(t)}\right)
\end{aligned}
$$

and hence, by (1.10),

$$
\begin{aligned}
\sum_{k=1}^{n} v_{k}\left(\mu_{k 1}+\tilde{l}_{k k}+l_{k k}\right) S_{k}^{*}\left(1-\frac{1}{x_{k}(t)}\right)\left(x_{k}(t)-1\right) \\
=\sum_{k=1}^{n} v_{k}\left(\mu_{k 1}+\tilde{l}_{k k}+l_{k k}\right) S_{k}^{*}\left\{g\left(x_{k}(t)\right)+g\left(\frac{1}{x_{k}(t)}\right)\right\}
\end{aligned}
$$


and

$$
\begin{aligned}
\sum_{k=1}^{n} v_{k} & \sum_{j=1}^{n} l_{k j} S_{j}^{*}\left(1-\frac{1}{x_{k}(t)}\right)\left(x_{j}(t-\sigma)-1\right) \\
& =\sum_{k=1}^{n} v_{k} \sum_{j=1}^{n} l_{k j} S_{j}^{*}\left\{g\left(x_{j}(t-\sigma)\right)-g\left(\frac{x_{j}(t-\sigma)}{x_{k}(t)}\right)+g\left(\frac{1}{x_{k}(t)}\right)\right\}
\end{aligned}
$$

For the remaining parts of the last equation in (5.3), we have

$$
\begin{aligned}
(1- & \left.\frac{1}{x_{k}(t)}\right)\left(1-x_{k}(t) \tilde{y}_{j}(t-\tau)\right)+\left(1-\frac{1}{y_{k}(t)}\right)\left(x_{k}(t) \tilde{y}_{j}(t-\tau)-y_{k}(t)\right) \\
= & \left(1-\frac{1}{x_{k}(t)}-x_{k}(t) \tilde{y}_{j}(t-\tau)+\tilde{y}_{j}(t-\tau)\right) \\
& +\left(x_{k}(t) \tilde{y}_{j}(t-\tau)-\frac{x_{k}(t) \tilde{y}_{j}(t-\tau)}{y_{k}(t)}-y_{k}(t)+1\right) \\
= & 2-\frac{1}{x_{k}(t)}+\tilde{y}_{j}(t-\tau)-\frac{x_{k}(t) \tilde{y}_{j}(t-\tau)}{y_{k}(t)}-y_{k}(t) \\
= & -g\left(\frac{1}{x_{k}(t)}\right)-g\left(\frac{x_{k}(t) \tilde{y}_{j}(t-\tau)}{y_{k}(t)}\right)+\left\{g\left(\tilde{y}_{j}(t-\tau)\right)-g\left(y_{k}(t)\right)\right\},
\end{aligned}
$$

and

$$
\begin{aligned}
\left(1-\frac{1}{y_{k}(t)}\right)\left(y_{j}(t-\sigma)-y_{k}(t)\right) & =y_{j}(t-\sigma)-\frac{y_{j}(t-\sigma)}{y_{k}(t)}-y_{k}(t)+1 \\
& =-g\left(\frac{y_{j}(t-\sigma)}{y_{k}(t)}\right)+\left\{g\left(y_{j}(t-\sigma)\right)-g\left(y_{k}(t)\right)\right\} .
\end{aligned}
$$

Then,

$$
\begin{aligned}
\sum_{k=1}^{n} v_{k}\left[\sum _ { j = 1 } ^ { n } \beta _ { k j } S _ { k } ^ { * } G ( I _ { j } ^ { * } ) \int _ { 0 } ^ { h _ { 1 } } f _ { k j } ( \tau ) \left\{\left(1-\frac{1}{x_{k}(t)}\right)\left(1-x_{k}(t) \tilde{y}_{j}(t-\tau)\right)\right.\right. \\
\left.+\left(1-\frac{1}{y_{k}(t)}\right)\left(x_{k}(t) \tilde{y}_{j}(t-\tau)-y_{k}(t)\right)\right\} d \tau \\
\left.+\left(1-\frac{1}{y_{k}(t)}\right) \sum_{j=1}^{n} m_{k j} I_{j}^{*} \int_{0}^{h_{2}} g_{k j}(\sigma)\left(y_{j}(t-\sigma)-y_{k}(t)\right) d \sigma\right] \\
=-\sum_{k=1}^{n} v_{k} \sum_{j=1}^{n}\left[\beta_{k j} S_{k}^{*} G\left(I_{j}^{*}\right)\left\{g\left(\frac{1}{x_{k}(t)}\right)+\int_{0}^{h_{1}} f_{k j}(\tau) g\left(\frac{x_{k}(t) \tilde{y}_{j}(t-\tau)}{y_{k}(t)}\right) d \tau\right\}\right. \\
\left.+m_{k j} I_{j}^{*} \int_{0}^{h_{2}} g_{k j}(\sigma) g\left(\frac{y_{j}(t-\sigma)}{y_{k}(t)}\right) d \sigma\right] \\
+\sum_{k=1}^{n} v_{k} \sum_{j=1}^{n}\left(\beta_{k j} S_{k}^{*} G\left(I_{j}^{*}\right) \int_{0}^{h_{1}} f_{k j}(\tau)\left\{g\left(\tilde{y}_{j}(t-\tau)\right)-g\left(y_{k}(t)\right)\right\} d \tau\right. \\
\left.+m_{k j} I_{j}^{*} \int_{0}^{h_{2}} g_{k j}(\sigma)\left\{g\left(y_{j}(t-\sigma)\right)-g\left(y_{k}(t)\right)\right\} d \sigma\right) .
\end{aligned}
$$


We finally obtain that

$$
\begin{aligned}
& \frac{d U(t)}{d t}=-\sum_{k=1}^{n} v_{k}\left(\mu_{k 1}+\tilde{l}_{k k}+l_{k k}\right) S_{k}^{*}\left\{g\left(x_{k}(t)\right)+g\left(\frac{1}{x_{k}(t)}\right)\right\} \\
& +\sum_{k=1}^{n} v_{k} \sum_{j=1}^{n} l_{k j} S_{j}^{*} \int_{0}^{h_{2}} g_{k j}(\sigma)\left\{g\left(x_{j}(t-\sigma)\right)-g\left(\frac{x_{j}(t-\sigma)}{x_{k}(t)}\right)+g\left(\frac{1}{x_{k}(t)}\right)\right\} d \sigma \\
& -\sum_{k=1}^{n} v_{k} \sum_{j=1}^{n}\left[\beta_{k j} S_{k}^{*} G\left(I_{j}^{*}\right)\left\{g\left(\frac{1}{x_{k}(t)}\right)+\int_{0}^{h_{1}} f_{k j}(\tau) g\left(\frac{x_{k}(t) \tilde{y}_{j}(t-\tau)}{y_{k}(t)}\right) d \tau\right\}\right. \\
& \left.+m_{k j} I_{j}^{*} \int_{0}^{h_{2}} g_{k j}(\sigma) g\left(\frac{y_{j}(t-\sigma)}{y_{k}(t)}\right) d \sigma\right] \\
& +\sum_{k=1}^{n} v_{k} \sum_{j=1}^{n}\left(\beta_{k j} S_{k}^{*} G\left(I_{j}^{*}\right) \int_{0}^{h_{1}} f_{k j}(\tau)\left\{g\left(\tilde{y}_{j}(t-\tau)\right)-g\left(y_{k}(t)\right)\right\} d \tau\right. \\
& \left.+m_{k j} I_{j}^{*} \int_{0}^{h_{2}} g_{k j}(\sigma)\left\{g\left(y_{j}(t-\sigma)\right)-g\left(y_{k}(t)\right)\right\} d \sigma\right) \\
& +\sum_{k=1}^{n} v_{k}\left(\sum_{j=1}^{n} \beta_{k j} S_{k}^{*} G\left(I_{j}^{*}\right) \int_{0}^{h_{1}} f_{k j}(\tau)\left\{g\left(\tilde{y}_{j}(t)\right)-g\left(\tilde{y}_{j}(t-\tau)\right)\right\} d \tau\right. \\
& +\sum_{j=1}^{n} l_{k j} S_{j}^{*} \int_{0}^{h_{2}} g_{k j}(\sigma)\left\{g\left(x_{j}(t)\right)-g\left(x_{j}(t-\sigma)\right)\right\} d \sigma \\
& \left.+\sum_{j=1}^{n} m_{k j} I_{j}^{*} \int_{0}^{h_{2}} g_{k j}(\sigma)\left\{g\left(y_{j}(t)\right)-g\left(y_{j}(t-\sigma)\right)\right\} d \sigma\right) \\
& =-\sum_{k=1}^{n} v_{k}\left(\mu_{k 1}+\tilde{l}_{k k}+l_{k k}\right) S_{k}^{*}\left\{g\left(x_{k}(t)\right)+g\left(\frac{1}{x_{k}(t)}\right)\right\} \\
& +\sum_{k=1}^{n} v_{k} \sum_{j=1}^{n} l_{k j} S_{j}^{*} \int_{0}^{h_{2}} g_{k j}(\sigma)\left\{g\left(x_{j}(t)\right)-g\left(\frac{x_{j}(t-\sigma)}{x_{k}(t)}\right)+g\left(\frac{1}{x_{k}(t)}\right)\right\} d \sigma \\
& -\sum_{k=1}^{n} v_{k} \sum_{j=1}^{n}\left[\beta_{k j} S_{k}^{*} G\left(I_{j}^{*}\right)\left\{g\left(\frac{1}{x_{k}(t)}\right)+\int_{0}^{h_{1}} f_{k j}(\tau) g\left(\frac{x_{k}(t) \tilde{y}_{j}(t-\tau)}{y_{k}(t)}\right) d \tau\right\}\right. \\
& \left.+m_{k j} I_{j}^{*} \int_{0}^{h_{2}} g_{k j}(\sigma) g\left(\frac{y_{j}(t-\sigma)}{y_{k}(t)}\right) d \sigma\right] \\
& +\sum_{k=1}^{n} v_{k} \sum_{j=1}^{n}\left(\beta_{k j} S_{k}^{*} G\left(I_{j}^{*}\right)\left\{g\left(\tilde{y}_{j}(t)\right)-g\left(y_{k}(t)\right)\right\}+m_{k j} I_{j}^{*}\left\{g\left(y_{j}(t)\right)-g\left(y_{k}(t)\right)\right\}\right) \\
& \leq-\sum_{k=1}^{n} v_{k}\left(\mu_{k 1}+\tilde{l}_{k k}+l_{k k}\right) S_{k}^{*}\left\{g\left(x_{k}(t)\right)+g\left(\frac{1}{x_{k}(t)}\right)\right\} \\
& +\sum_{k=1}^{n} v_{k} \sum_{j=1}^{n} l_{k j} S_{j}^{*} \int_{0}^{h_{2}} g_{k j}(\sigma)\left\{g\left(x_{j}(t)\right)-g\left(\frac{x_{j}(t-\sigma)}{x_{k}(t)}\right)+g\left(\frac{1}{x_{k}(t)}\right)\right\} d \sigma \\
& -\sum_{k=1}^{n} v_{k} \sum_{j=1}^{n}\left[\beta_{k j} S_{k}^{*} G\left(I_{j}^{*}\right)\left\{g\left(\frac{1}{x_{k}(t)}\right)+\int_{0}^{h_{1}} f_{k j}(\tau) g\left(\frac{x_{k}(t) \tilde{y}_{j}(t-\tau)}{y_{k}(t)}\right) d \tau\right\}\right. \\
& \left.+m_{k j} I_{j}^{*} \int_{0}^{h_{2}} g_{k j}(\sigma) g\left(\frac{y_{j}(t-\sigma)}{y_{k}(t)}\right) d \sigma\right] \\
& +\sum_{k=1}^{n} v_{k} \sum_{j=1}^{n}\left(\beta_{k j} S_{k}^{*} G\left(I_{j}^{*}\right)\left\{g\left(y_{j}(t)\right)-g\left(y_{k}(t)\right)\right\}+m_{k j} I_{j}^{*}\left\{g\left(y_{j}(t)\right)-g\left(y_{k}(t)\right)\right\}\right) .
\end{aligned}
$$


Here, we use the relation $g\left(\tilde{y}_{j}(t)\right) \leq g\left(y_{j}(t)\right), j=1,2, \ldots, n$ (see Enatsu et al. [6, Lemma 3.2]), because $y_{j}(t)=I_{j}(t) / I_{j}^{*} \geq 1$ if and only if

$$
y_{j}(t)-\tilde{y}_{j}(t)=\frac{I_{j}(t)}{I_{j}^{*}}-\frac{G\left(I_{j}(t)\right)}{G\left(I_{j}^{*}\right)}=\frac{G\left(I_{j}(t)\right)}{I_{j}^{*}}\left(\frac{I_{j}(t)}{G\left(I_{j}(t)\right)}-\frac{I_{j}^{*}}{G\left(I_{j}^{*}\right)}\right) \geq 0,
$$

which yields

$$
\begin{aligned}
\frac{d U(t)}{d t} \leq & -\sum_{k=1}^{n} v_{k}\left(\mu_{k 1}+\tilde{l}_{k k}+l_{k k}\right) S_{k}^{*}\left\{g\left(x_{k}(t)\right)+g\left(\frac{1}{x_{k}(t)}\right)\right\} \\
& +\sum_{k=1}^{n} v_{k} \sum_{j=1}^{n} l_{k j} S_{j}^{*} \int_{0}^{h_{2}} g_{k j}(\sigma)\left\{g\left(x_{j}(t)\right)-g\left(\frac{x_{j}(t-\sigma)}{x_{k}(t)}\right)+g\left(\frac{1}{x_{k}(t)}\right)\right\} d \sigma \\
& -\sum_{k=1}^{n} v_{k} \sum_{j=1}^{n}\left[\beta_{k j} S_{k}^{*} G\left(I_{j}^{*}\right)\left\{g\left(\frac{1}{x_{k}(t)}\right)+\int_{0}^{h_{1}} f_{k j}(\tau) g\left(\frac{x_{k}(t) \tilde{y}_{j}(t-\tau)}{y_{k}(t)}\right) d \tau\right\}\right. \\
& \left.\quad+m_{k j} I_{j}^{*} \int_{0}^{h_{2}} g_{k j}(\sigma) g\left(\frac{y_{j}(t-\sigma)}{y_{k}(t)}\right) d \sigma\right] \\
& +\sum_{k=1}^{n} v_{k} \sum_{j=1}^{n}\left(\beta_{k j} S_{k}^{*} \frac{G\left(I_{j}^{*}\right)}{I_{j}^{*}}+\left(1-\delta_{k j}\right) m_{k j}\right) I_{j}^{*}\left\{g\left(y_{j}(t)\right)-g\left(y_{k}(t)\right)\right\} .
\end{aligned}
$$

On the other hand, by (4.1), we have that

$$
\begin{aligned}
\sum_{k=1}^{n} v_{k} & \sum_{j=1}^{n}\left(\beta_{k j} S_{k}^{*} \frac{G\left(I_{j}^{*}\right)}{I_{j}^{*}}+\left(1-\delta_{k j}\right) m_{k j}\right) I_{j}^{*}\left\{g\left(y_{j}(t)\right)-g\left(y_{k}(t)\right)\right\} \\
= & \sum_{k=1}^{n} v_{k} \sum_{j=1}^{n}\left(\beta_{k j} S_{k}^{*} \frac{G\left(I_{j}^{*}\right)}{I_{j}^{*}}+\left(1-\delta_{k j}\right) m_{k j}\right) I_{j}^{*} g\left(y_{j}(t)\right) \\
& -\sum_{k=1}^{n} v_{k}\left\{\sum_{j=1}^{n}\left(\beta_{k j} S_{k}^{*} \frac{G\left(I_{j}^{*}\right)}{I_{j}^{*}}+\left(1-\delta_{k j}\right) m_{k j}\right) I_{j}^{*}\right\} g\left(y_{k}(t)\right) \\
= & \sum_{j=1}^{n} v_{j} \sum_{k=1}^{n}\left(\beta_{j k} S_{j}^{*} \frac{G\left(I_{j}^{*}\right)}{I_{j}^{*}}+\left(1-\delta_{j k}\right) m_{j k}\right) I_{k}^{*} g\left(y_{k}(t)\right)-\sum_{k=1}^{n} v_{k}\left(\mu_{k 2}+\gamma_{k}+\tilde{m}_{k k}\right) I_{k}^{*} g\left(y_{k}(t)\right) \\
= & \sum_{k=1}^{n}\left\{\sum_{j=1}^{n} v_{j}\left(\beta_{j k} S_{j}^{*} \frac{G\left(I_{j}^{*}\right)}{I_{j}^{*}}+\left(1-\delta_{j k}\right) m_{j k}\right)-v_{k}\left(\mu_{k 2}+\gamma_{k}+\tilde{m}_{k k}\right)\right\} I_{k}^{*} g\left(y_{k}(t)\right) .
\end{aligned}
$$

From (5.5) and (5.6), we therefore obtain that

$$
\begin{aligned}
\frac{d U(t)}{d t} \leq & -\sum_{k=1}^{n} v_{k}\left(\mu_{k 1}+\tilde{l}_{k k}+l_{k k}\right) S_{k}^{*}\left\{g\left(x_{k}(t)\right)+g\left(\frac{1}{x_{k}(t)}\right)\right\} \\
& +\sum_{k=1}^{n} v_{k} \sum_{j=1}^{n} l_{k j} S_{j}^{*} \int_{0}^{h_{2}} g_{k j}(\sigma)\left\{g\left(x_{j}(t)\right)-g\left(\frac{x_{j}(t-\sigma)}{x_{k}(t)}\right)+g\left(\frac{1}{x_{k}(t)}\right)\right\} d \sigma \\
& -\sum_{k=1}^{n} v_{k} \sum_{j=1}^{n}\left[\beta_{k j} S_{k}^{*} G\left(I_{j}^{*}\right)\left\{g\left(\frac{1}{x_{k}(t)}\right)+\int_{0}^{h_{1}} f_{k j}(\tau) g\left(\frac{x_{k}(t) \tilde{y}_{j}(t-\tau)}{y_{k}(t)}\right) d \tau\right\}\right. \\
& \left.\quad+m_{k j} I_{j}^{*} \int_{0}^{h_{2}} g_{k j}(\sigma) g\left(\frac{y_{j}(t-\sigma)}{y_{k}(t)}\right) d \sigma\right] \\
& +\sum_{k=1}^{n}\left\{\sum_{j=1}^{n} v_{j}\left(\beta_{j k} S_{j}^{*} \frac{G\left(I_{j}^{*}\right)}{I_{j}^{*}}+\left(1-\delta_{j k}\right) m_{j k}\right)-v_{k}\left(\mu_{k 2}+\gamma_{k}+\tilde{m}_{k k}\right)\right\} I_{k}^{*} g\left(y_{k}(t)\right)
\end{aligned}
$$




$$
\begin{aligned}
= & -\sum_{k=1}^{n}\left\{v_{k}\left(\mu_{k 1}+\tilde{l}_{k k}+l_{k k}\right)-\sum_{j=1}^{n} v_{j} l_{j k}\right\} S_{k}^{*} g\left(x_{k}(t)\right) \\
& -\sum_{k=1}^{n} v_{k}\left\{\left(\sum_{j=1}^{n} \beta_{k j} G\left(I_{j}^{*}\right)+\left(\mu_{k 1}+\tilde{l}_{k k}+l_{k k}\right) S_{k}^{*}-\sum_{j=1}^{n} l_{k j} S_{j}^{*}\right\} g\left(\frac{1}{x_{k}(t)}\right)\right. \\
& -\sum_{k=1}^{n} v_{k} \sum_{j=1}^{n} l_{k j} S_{j}^{*} \int_{0}^{h_{2}} g_{k j}(\sigma) g\left(\frac{x_{j}(t-\sigma)}{x_{k}}\right) d \sigma \\
& -\sum_{k=1}^{n} v_{k} \sum_{j=1}^{n}\left\{\beta_{k j} S_{k}^{*} G\left(I_{j}^{*}\right) \int_{0}^{h_{1}} f_{k j}(\tau)\left(\frac{x_{k}(t) \tilde{y}_{j}(t-\tau)}{y_{k}(t)}\right) d \tau\right. \\
& \left.\quad+m_{k j} I_{j}^{*} \int_{0}^{h_{2}} g_{k j}(\sigma) g\left(\frac{y_{j}(t-\sigma)}{y_{k}(t)}\right) d \sigma\right\} \\
& +\sum_{k=1}^{n}\left\{\sum_{j=1}^{n} v_{j}\left(\beta_{j k} S_{j}^{*} \frac{G\left(I_{j}^{*}\right)}{I_{j}^{*}}+\left(1-\delta_{j k}\right) m_{j k}\right)-v_{k}\left(\mu_{k 2}+\gamma_{k}+\tilde{m}_{k k}\right)\right\} I_{k}^{*} g\left(y_{k}(t)\right) .
\end{aligned}
$$

Hence, by (4.1),

$$
\begin{aligned}
\frac{d U}{d t} \leq & -\sum_{k=1}^{n}\left\{v_{k}\left(\mu_{k 1}+\tilde{l}_{k k}+l_{k k}\right)-\sum_{j=1}^{n} v_{j} l_{j k}\right\} S_{k}^{*} g\left(x_{k}(t)\right) \\
& -\sum_{k=1}^{n} v_{k} b_{k} g\left(\frac{1}{x_{k}(t)}\right)-\sum_{k=1}^{n} v_{k} \sum_{j=1}^{n} l_{k j} S_{j}^{*} \int_{0}^{h_{2}} g_{k j}(\sigma) g\left(\frac{x_{j}(t-\sigma)}{x_{k}}\right) d \sigma \\
& -\sum_{k=1}^{n} v_{k} \sum_{j=1}^{n}\left\{\beta_{k j} S_{k}^{*} G\left(I_{j}^{*}\right) \int_{0}^{h_{1}} f_{k j}(\tau)\left(\frac{x_{k}(t) \tilde{y}_{j}(t-\tau)}{y_{k}(t)}\right) d \tau\right. \\
& \left.\quad+m_{k j} I_{j}^{*} \int_{0}^{h_{2}} g_{k j}(\sigma) g\left(\frac{y_{j}(t-\sigma)}{y_{k}(t)}\right) d \sigma\right\} \\
& +\sum_{k=1}^{n}\left\{\sum_{j=1}^{n} v_{j}\left(\beta_{j k} S_{j}^{*} \frac{G\left(I_{j}^{*}\right)}{I_{j}^{*}}+\left(1-\delta_{j k}\right) m_{j k}\right)-v_{k}\left(\mu_{k 2}+\gamma_{k}+\tilde{m}_{k k}\right)\right\} I_{k}^{*} g\left(y_{k}(t)\right),
\end{aligned}
$$

from which one can obtain the following lemma.

Lemma 5.1. For $\tilde{R}_{0}>1$, if there exists a positive $n$-column vector $\mathbf{v}=\left(v_{1}, v_{2}, \ldots, v_{n}\right)^{T}$ such that (1.17) holds, then $U^{\prime}(t) \leq 0$.

Proof of Theorem 1.1. Theorem 3.1 denotes the first part $\tilde{R}_{0} \leq 1$ of Theorem 1.1. For the case $\tilde{R}_{0}>1$, by Lemma 4.1, there exists at least one endemic equilibrium $\mathbf{E}^{*}=\left(S_{1}^{*}, I_{1}^{*}, S_{2}^{*}, I_{2}^{*}, \ldots\right.$, $\left.S_{n}^{*}, I_{n}^{*}\right)$ and by Lemmas 2.1 and 4.4, system (1.6) is permanent in $\Gamma^{0}$. We now suppose that there exists a positive $n$-column vector $\mathbf{v}$ such that (1.17) holds. By Lemma 5.1, it hold (5.7) for (5.2) and $U^{\prime}(t)=0$, if and only if

$$
x_{k}(t)=1 \text {, and } y_{k}(t)=y_{j}(t-\sigma) \text {, for any } 0 \leq \sigma \leq h_{2}, t>0, j=1,2, \ldots, n, k=1,2, \ldots, n .
$$

Then, there exists a positive constant $c$ such that

$$
\frac{I_{k}(t)}{I_{k}^{*}}=c, \quad \text { for any } t>0, j=1,2, \ldots, n, k=1,2, \ldots, n,
$$


and

$$
S_{k}(t)=S_{k}^{*} \quad \text { and } \quad I_{k}(t)=c I_{k}^{*}, \quad \text { for any } t>0, k=1,2, \ldots, n .
$$

From the first equation of system (1.6), we obtain that

$$
0=b_{k}-\left(\mu_{k 1}+\tilde{l}_{k k}+l_{k k}\right)+c \sum_{j=1}^{n} \beta_{k j} S_{k}^{*} G\left(I_{j}^{*}\right)-l_{k j} S_{j}^{*}, \quad \text { for any } k=1,2, \ldots, n .
$$

Since the right-hand side of (5.8) is strictly decreasing in $c$, (5.8) holds if and only if $c=1$. This implies that $\left(S_{1}(t), I_{1}(t), S_{2}(t), I_{2}(t), \ldots, S_{n}(t), I_{n}(t)\right)=\mathbf{E}^{*}$. Then, the only compact invariant subset where $U^{\prime}(t)=0$ is the singleton $\left\{\mathbf{E}^{*}\right\}$. Therefore, by Lemmas 2.1, 2.2 and 4.4, and a similar argument as in Section 3 , we obtain that for $\tilde{R}_{0}>1, \mathbf{E}^{*}$ is globally asymptotically stable in $\Gamma^{0}$. Hence, the proof is completed.

Concerning condition (1.18), we have the following lemma (see Guo et al. [8, Lemma 2.1]).

Lemma 5.2. The following system:

$$
\sum_{j=1}^{n} v_{j}\left\{\beta_{j k} S_{j}^{*} \frac{G\left(I_{j}^{*}\right)}{I_{j}^{*}}+\left(1-\delta_{j k}\right) m_{j k}\right\}=v_{k}\left(\mu_{k 2}+\gamma_{k}+\tilde{m}_{k k}\right), \quad k=1,2, \ldots, n
$$

has a positive solution $\left(v_{1}, v_{2}, \ldots, v_{n}\right)$ defined by

$$
\left(v_{1}, v_{2}, \cdots, v_{n}\right)=\left(C_{11}, C_{22}, \ldots, C_{n n}\right),
$$

where

$$
\tilde{\beta}_{k j}=\left(\beta_{k j} S_{k}^{*} \frac{G\left(I_{j}^{*}\right)}{I_{j}^{*}}+\left(1-\delta_{k j}\right) m_{k j}\right) I_{j}^{*}, \quad 1 \leq k, j \leq n,
$$

and

$$
\tilde{\mathbf{B}}=\left[\begin{array}{cccc}
\sum_{j \neq 1} \tilde{\beta}_{1 j} & -\tilde{\beta}_{21} & \cdots & -\tilde{\beta}_{n 1} \\
-\tilde{\beta}_{12} & \sum_{j \neq 2} \tilde{\beta}_{2 j} & \cdots & -\tilde{\beta}_{n 2} \\
\vdots & \vdots & \ddots & \vdots \\
-\tilde{\beta}_{1 n} & -\tilde{\beta}_{2 n} & \cdots & \sum_{j \neq n} \tilde{\beta}_{n j}
\end{array}\right],
$$

and $C_{k k}$ denotes the cofactor of the $k$-th diagonal entry of $\tilde{\mathbf{B}}, 1 \leq k \leq n$.

Proof. Consider a basis for the solution space of the linear system:

$$
\tilde{\mathbf{B}} \mathbf{v}=0,
$$

which can be written as (5.10) (see for example, Berman and Plemmons [3]). By the irreducibility of $\mathbf{B}$, one can see that $\left[\tilde{\beta}_{k j}\right]_{n \times n}$ is irreducible and $v_{k}=C_{k k}>0, k=1,2, \ldots, n$. Then, by (5.11), we have that

$$
\left[\begin{array}{cccc}
\tilde{\beta}_{11} & \tilde{\beta}_{21} & \cdots & \tilde{\beta}_{n 1} \\
\tilde{\beta}_{12} & \tilde{\beta}_{22} & \cdots & \tilde{\beta}_{n 2} \\
\vdots & \vdots & \ddots & \vdots \\
\tilde{\beta}_{1 n} & \tilde{\beta}_{2 n} & \cdots & \tilde{\beta}_{n n}
\end{array}\right]\left[\begin{array}{c}
v_{1} \\
v_{2} \\
\vdots \\
v_{n}
\end{array}\right]=\left[\begin{array}{c}
\left(\sum_{j=1}^{n} \tilde{\beta}_{1 j}\right) v_{1} \\
\left(\sum_{j=1}^{n} \tilde{\beta}_{2 j}\right) v_{2} \\
\vdots \\
\left(\sum_{j=1}^{n} \tilde{\beta}_{n j}\right) v_{n}
\end{array}\right],
$$


from which we have that

$$
\sum_{j=1}^{n} v_{j} \tilde{\beta}_{j k}=v_{k} \sum_{j=1}^{n} \tilde{\beta}_{k j}, \quad k=1,2, \ldots, n,
$$

which is equivalent to

$$
\begin{aligned}
\sum_{j=1}^{n} v_{j}\left\{\beta_{j k} S_{j}^{*} \frac{G\left(I_{j}^{*}\right)}{I_{j}^{*}}+\left(1-\delta_{j k}\right) m_{j k}\right\} I_{k}^{*} & =v_{k} \sum_{j=1}^{n}\left\{\beta_{k j} S_{k}^{*} \frac{G\left(I_{k}^{*}\right)}{I_{k}^{*}}+\left(1-\delta_{k j}\right) m_{k j}\right\} I_{j}^{*} \\
& =v_{k}\left(\mu_{k 2}+\gamma_{k}+\tilde{m}_{k k}\right) I_{k}^{*},
\end{aligned}
$$

for $k=1,2, \ldots, n$. By $I_{k}^{*}>0$, one can see that (5.9) has a positive solution $\left(v_{1}, v_{2}, \ldots, v_{n}\right)$ defined by (5.10).

Proof of Corollary 1.2. For system (1.6), it follows from Lemma 5.2 that there exists a positive $n$-column vector $\mathbf{v}=\left(v_{1}, v_{2}, \ldots, v_{n}\right)^{T}$ such that (1.18) and (1.19) hold. By Theorem 1.1, we obtain the conclusion of the first part of this corollary. If there exists positive $n$-column vector $\mathbf{w}=\left(w_{1}, w_{2}, \ldots, w_{n}\right)^{T}$ such that (1.20) and (1.21) hold, then by Theorem 1.1, we obtain the conclusion of the second part of this corollary.

By Corollary 1.2, we obtain the following corollaries.

Corollary 5.3. If $\tilde{R}_{0} \leq 1$ and

$$
\left\{\begin{array}{l}
\mu_{k 1}+\tilde{l}_{k k} \geq \mu_{k 2}+\gamma_{k}+\tilde{m}_{k k}, \quad \text { and } \\
\beta_{j k} S_{j}^{0}+\left(1-\delta_{j k}\right) m_{j k} \geq\left(1-\delta_{j k}\right) l_{j k}, \text { for any } j, k=1,2, \ldots, n, \\
o r, \\
\mu_{k 1}+\tilde{l}_{k k} \leq \mu_{k 2}+\gamma_{k}+\tilde{m}_{k k}, \quad \text { and } \\
\beta_{j k} S_{j}^{0}+\left(1-\delta_{j k}\right) m_{j k} \leq\left(1-\delta_{j k}\right) l_{j k}, \text { for any } j, k=1,2, \ldots, n,
\end{array}\right.
$$

then $\mathbf{E}^{0}$ is globally asymptotically stable in $\Gamma$.

If $\tilde{R}_{0}>1$ and

$$
\left\{\begin{array}{l}
\mu_{k 1}+\tilde{l}_{k k} \geq \mu_{k 2}+\gamma_{k}+\tilde{m}_{k k}, \quad \text { and } \\
\beta_{j k} S_{j}^{*} \frac{G\left(I_{k}^{*}\right)}{I_{k}^{*}}+\left(1-\delta_{j k}\right) m_{j k} \geq\left(1-\delta_{j k}\right) l_{j k}, \text { for any } j, k=1,2, \ldots, n, \\
o r, \\
\mu_{k 1}+\tilde{l}_{k k} \leq \mu_{k 2}+\gamma_{k}+\tilde{m}_{k k}, \quad \text { and } \\
\beta_{j k} S_{j}^{*} \frac{G\left(I_{k}^{*}\right)}{I_{k}^{*}}+\left(1-\delta_{j k}\right) m_{j k} \leq\left(1-\delta_{j k}\right) l_{j k}, \text { for any } j, k=1,2, \ldots, n
\end{array}\right.
$$

then $\mathbf{E}^{*}$ is globally asymptotically stable in $\boldsymbol{\Gamma}^{0}$.

Corollary 5.4. If

$$
\left\{\begin{array}{l}
\mu_{k 1}+\tilde{l}_{k k} \geq \mu_{k 2}+\gamma_{k}+\tilde{m}_{k k}, \quad \text { and } \\
m_{j k} \geq l_{j k}, \text { for any } j \neq k, k=1,2, \ldots, n,
\end{array}\right.
$$


then for $\tilde{R}_{0} \leq 1, \mathbf{E}^{0}$ is globally asymptotically stable in $\boldsymbol{\Gamma}$, and for $\tilde{R}_{0}>1, \mathbf{E}^{*}$ is globally asymptotically stable in $\Gamma^{0}$.

If

$$
\left\{\begin{array}{l}
\mu_{k 1}+\tilde{l}_{k k} \leq \mu_{k 2}+\gamma_{k}+\tilde{m}_{k k}, \quad \text { and } \\
\beta_{j k} S_{j}^{0}+\left(1-\delta_{j k}\right) m_{j k} \leq\left(1-\delta_{j k}\right) l_{j k}, \text { for any } j, k=1,2, \ldots, n
\end{array}\right.
$$

then for $\tilde{R}_{0} \leq 1, \mathbf{E}^{0}$ is globally asymptotically stable in $\boldsymbol{\Gamma}$, and for $\tilde{R}_{0}>1, \mathbf{E}^{*}$ is globally asymptotically stable in $\Gamma^{0}$, where $\bar{I}_{k}^{*}, k=1,2, \ldots, n$ are determined by the following equation.

$$
\left(\mu_{k 2}+\gamma_{k}+\tilde{m}_{k k}\right) \bar{I}_{k}^{*}=\sum_{j=1}^{n}\left\{\beta_{k j} S_{k}^{0}+\left(1-\delta_{k j}\right) m_{k j}\right\} \bar{I}_{j}^{*}, \quad k=1,2, \ldots, n
$$

Note that $G\left(I_{k}^{*}\right)<\bar{I}_{k}^{*}, k=1,2, \ldots, n$, if $I_{k}^{*}>0, k=1,2, \ldots, n$ exist (see (4.1)).

\section{Discussion}

In this paper, we have formulated the multi-group SIR epidemic model (1.4) with nonlinear incidence rates and distributed delays, which is related to the heroin model in which the heroin users under treatment can return to untreated users depending on their different characters and external influences. We have simplified the model to (1.6) and investigated the global asymptotic stability of its equilibria. We have defined the threshold parameter $\tilde{R}_{0}$ as in (1.13) and proved that if $\tilde{R}_{0}<1$ and there exists a positive $n$-column vector $\mathbf{u}$ such that (1.16) holds, then the disease-free equilibrium $\mathbf{E}^{0}$ is globally asymptotically stable, while if $\tilde{R}_{0}>1$ and there exists a positive $n$-column vector $\mathbf{v}$ such that (1.17) holds, then the endemic equilibrium $\mathbf{E}^{*}$ is globally asymptotically stable (see Theorem 1.1).

The condition (5.14) in Corollary 5.4, as well as the latter part of the conditions (5.12) and (5.13) in Corollary 5.3, implies that $\beta_{k k}=0$ for all $k=1,2, \ldots, n$. It is worth noting that the condition $\beta_{k k}=0$ for all $k=1,2, \ldots, n$ is valid, since the matrix $\mathbf{B}$ is assumed to be irreducible. For the case $\mu_{k 1}+\tilde{l}_{k k} \leq \mu_{k 2}+\gamma_{k}+\tilde{m}_{k k}$ and $\beta_{k k}>0$ for some $k=1,2, \ldots, n$, it would be an interesting open problem to find the sufficient conditions under which each of equilibria $\mathbf{E}^{0}$ and $\mathbf{E}^{*}$ is globally stable.

\section{Acknowledgements}

The authors express their deep gratitude to Professor Dr. Tibor Krisztin and the members of the organizing committee in charge of "10th Colloquium on the Qualitative Theory of Differential Equations". The authors also would like to thank the editors and the anonymous reviewer for his/her valuable comments to the previous version of this paper. YM was supported by Scientific Research (c), No. 15K05010 of Japan Society for the Promotion of Science. TK was supported by Grant-in-Aid for Young Scientists (B), No. 15K17585 of Japan Society for the Promotion of Science and the program of the Japan Agency for Medical Research and Development, AMED. YE was supported by Grant-in-Aid for Young Scientists (B), No. 26800066 of Japan Society for the Promotion of Science. 


\section{References}

[1] J. ARINo, Diseases in metapopulations, in: Modeling and dynamics of infectious diseases, Higher Education Press, Beijing, 2009, 65-123. MR2523397

[2] M. S. Bartlet, Deterministic and stochastic models for recurrent epidemics, in: Proceedings of the Third Berkeley Symposium on Mathematical Statistics and Probability, University of California Press, California, 1956, 81-109. MR0084932

[3] A. Berman, R. J. Plemmons, Nonnegative matrices in the mathematical sciences, Academic Press, New York, 1979. MR1298430

[4] H. Chen, J. Sun, Global stability of delay multigroup epidemic models with group mixing nonlinear incidence rates, Appl. Math. Comput. 218(2011) 4391-4400. MR2862109

[5] O. Diekmann, J. A. P. Heesterbeek, Mathematical epidemiology of infectious diseases, Wiley, Chichester, UK, 2000. MR1882991

[6] Y. Enatsu, Y. NaKata, Y. Muroya, Lyapunov functional techniques for the global stability analysis of a delayed SIRS epidemic model, Nonlinear Anal. 13(2012), 2120-2133. MR2911901

[7] B. Fang, X. Li, M. Martcheva, L. CaI, Global stability for a heroin model with two distributed delays, Discrete Cont. Dynamic. Syst. Ser. B 19(2014), 715-733. MR3180723

[8] H. Guo, M. Y LI, Z. SHuAI, Global stability of the endemic equilibrium of multigroup SIR epidemic models, Can. Appl. Math. Q. 14(2006), 259-284. MR2327745

[9] H. Guo, M. Y LI, Global dynamics of a staged progression model with amelioration for infectious diseases, J. Biol. Dyn. 2(2008), 154-168. MR2427524

[10] H. Guo, M. Y LI, Z. SHUAI, A graph-theoretic approach to the method of global Lyapunov functions, Proc. Amer. Math. Soc. 136(2008), 2793-2802. MR2399043

[11] H. Guo, M. Y. LI, Global dynamics of a stage-progression model for HIV/AIDS with amelioration, Nonlinear Anal. 12(2011), 2529-2540. MR2813199

[12] G. HuANG, A. Liu, A note on global stability for a heroin epidemic model with distributed delay, Appl. Math. Lett. 26(2013), 687-691. MR3046379

[13] J. P. LASAlle, The stability of dynamical systems, SIAM, Philadelphia, 1976.

[14] J. LI, Y. XIAO, F. ZhanG, Y. YANG, An algebraic approach to proving the global stability of a class of epidemic models, Nonlinear Anal. 13(2012), 2006-2016. MR2911893

[15] M. Y. LI, Z. SHUAI, Global-stability problem for coupled systems of differential equations on networks, J. Differential Equations 284(2010), 1-20. MR2557892

[16] M. Y. LI, Z. ShUAI, C. WANG, Global stability of multi-group epidemic models with distributed delays, J. Math. Anal. Appl. 361(2010), 38-47. MR2567280

[17] J. Liu, T. ZhANG, Global behaviour of a heroin epidemic model with distributed delays, Appl. Math. Lett. 24(2011), 1685-1692. MR2803007 
[18] J. LiU, Y. ZHou, Global stability of an SIRS epidemic model with transport-related infection, Chaos Solitons Fractals 40(2009), 145-158. MR2517923

[19] X. LiU, Y. TAKeUchI, Spread of disease with transport-related infection, J. Theoret. Biol. 242(2006), 517-528. MR2272571

[20] T. Kajiwara, T. Sasaki, Y. Takeuchi, Construction of Lyapunov functionals for delay differential equations in virology and epidemiology, Nonlinear Anal. 13(2012), 1802-1826. MR2891011

[21] C. C. McCluskeY, Complete global stability for an SIR epidemic model with delayDistributed or discrete, Nonlinear Anal. 11(2010), 55-59. MR2570523

[22] G. Mulone, B. Straughan, A note on heroin epidemics, Math. Biosci. 218(2009), 138-141. MR2513678

[23] Y. Muroya, Practical monotonous iterations for nonlinear equations, Mem. Fac. Sci. Kyushu Univ. Ser. A 22(1968), 56-73. MR0229385

[24] Y. Muroya, Y. Enatsu, T. KuniYa, Global stability of extended multi-group SIR epidemic models with patches through migration and cross patch infection, Acta Math. Sci. Ser. B Engl. Ed. 33(2013), 341-361. MR3030623

[25] Y. Muroya, H. LI, T. KunIYA, Complete global analysis of an SIRS epidemic model with graded cure rate and incomplete recovery rate, J. Math. Anal. Appl. 410(2014), 719-732. MR3111862

[26] Y. NAKATA, On the global stability of a delayed epidemic model with transport-related infection, Nonlinear Anal. 12(2011), 3028-3034. MR2832945

[27] J. Ortega, W. Rheinboldt, Monotone iterations for nonlinear equations with application to Gauss-Seidel methods, SIAM J. Numer. Anal. 4(1967), 171-190. MR0215487

[28] G. P. Samanta, Dynamic behaviour for a nonautonomous heroin epidemic model with time delay, J. Appl. Math. Comput. 35(2011), 161-178. MR2748357

[29] H. SHU, D. FAN, J. WeI, Global stability of multi-group SEIR epidemic models with distributed delays and nonlinear transmission, Nonlinear Anal. 13(2012), 1581-1592. MR2890994

[30] R. Sun, Global stability of the endemic equilibrium of multigroup SIR models with nonlinear incidence, Comput. Math. Appl. 60(2010), 2286-2291. MR2725319

[31] R. S. VARgA, Matrix iterative analysis, Prentice-Hall, Inc. Englewood Cliffs, NJ, 1962. MR1753713

[32] C. VARgas-De-León, On the global stability of SIS, SIR and SIRS epidemic models, Chaos Solitons Fractals 44(2011), 1106-1110. url

[33] W. Wang, X. Zhao, An epidemic model in a patchy environment, Math. Bioci. 190(2004), 97-112. MR2067829

[34] X. WANG, J. YANG, X. LI, Dynamics of a heroin epidemic model with very population, Appl. Math. 2(2011), 732-738. MR2910185 
[35] E. White, C. Comiskey, Heroin epidemics, treatment and ODE modeling, Math. Biosci. 208(2007), 312-324. MR2330946

[36] Z. YUAN, L. WANG, Global stability of epidemiological models with group mixing and nonlinear incidence rates, Nonlinear Anal. 11(2010), 995-1004. MR2571270

[37] Z. YUAN, X. Zou, Global threshold property in an epidemic models for disease with latency spreading in a heterogeneous host population, Nonlinear Anal. 11(2010), 34793490. MR2683806 